\title{
MALACOFAUNA CONTINENTAL HOLOCENA (REGIÓN PAMPEANA, ARGENTINA)
}

\author{
PAMELA G. STEFFAN \\ Facultad de Ciencias Sociales, Universidad Nacional del Centro de la Provincia de Buenos Aires, Departamento de \\ Arqueología, INCUAPA-CONICET, Av. Del Valle 5737, 7400, Olavarría, Argentina.psteffan@soc.unicen.edu.ar
}

\section{MARINA L. AGUIRRE}

Facultad de Ciencias Naturales y Museo, Universidad Nacional de La Plata, Edificio Institutos, Laboratorios y Cátedras, CONICET, calle $64 \mathrm{~N}^{\circ}$ 3, 1900, La Plata, Argentina.maguirre@fcnym.unlp.edu.ar

\author{
SERGIO E. MIQUEL \\ Museo Argentino de Ciencias Naturales "Bernardino Rivadavia”, CONICET, Av. Ángel Gallardo 470, 1405, \\ Ciudad Autónoma de Buenos Aires, Argentina. semsnail@yahoo.com.ar
}

\begin{abstract}
HOLOCENE CONTINENTAL MOLLUSCAN FAUNA (PAMPEAN REGION, ARGENTINA). In this study the molluscan fauna recovered from six archaeological sites of the Interserrana Bonaerense area is analyzed. Three geographical sectors were studied in this area: I) Middle river basin of Quequén Grande river; II) Southwest of the Interserrana area, and III) Upper river basin of the Tapalqué stream. The systematic review of the continental mollusks recovered was complemented with quantitative, stratigraphical and chronological data for each site since the early Holocene until the present. Additionally, ecological requirements and modern distribution patterns of the taxa recorded were considered in order to search for eventual palaeoenvironmental variations in space and time. A total of 15 taxa (14 Gastropoda and one Bivalvia) were identified in the Luján Formation deposits from southeastern Buenos Aires Province. The first Holocene records for "Aplexa" minor (d'Orbigny), Drepanotrema kermatoides (d'Orbigny), Antillorbis nordestensis (Lucena), Omalonyx unguis d'Orbigny and Pisidium sp. are reported in the Bonaerese Interserrana area. Overall, the mollusks suggest palustrine habitats at the beginning of the early Holocene. During the middle Holocene, they suggest higher humidity (increase of the precipitation pattern) ca. 4.9 kyrs BP and ca. 4.7 kyrs BP. At the beginning of the late Holocene they indicate a general arid trend interrupted by brief humid episodes registered $c a$. 2.5 kyrs BP and $c a$. 2.2 kyrs BP. The integration and correlation of molluscan records in spatial and temporal scales allowed recognizing geographical displacements (i.e. Retidiscus reticulatus Fonseca \& Thomé) which represent biotic responses to Holocene climate variations as well as local environmental differences for stratigraphical sequences of similar chronologies.
\end{abstract}

Key words: continental mollusks, contexts of depositation, paleoenvironments, Late Quaternary, Interserrana Bonaerense area, Luján Formation.

RESUMO - No presente estudo é analisada a malacofauna proveniente de seis sítios arqueológicos da área Interserrana Bonaerense. Foram estudados três setores geográficos desta área: I) bacia média do río Quequén Grande; II) sudoeste da área Interserrana e III) bacia superior do arroio Tapalqué. A revisão sistemática dos moluscos continentais recuperados foi complementada com dados quantitativos, estratigráficos e cronológicos de cada sítio desde o Holoceno inicial até a atualidade. Adicionalmente, foram considerados os requerimentos ecológicos e os padrões de distribuição moderna dos táxons a fim de inferir eventuais variações paleoambientais. Um total de 15 táxons (14 Gastropoda e um Bivalvia) foram identificados nos depósitos da Formação Luján do sudeste da Província de Buenos Aires. Se dão a conhecer os primeiros registros holocênicos de "Aplexa" minor (d'Orbigny), Drepanotrema kermatoides (d'Orbigny), Antillorbis nordestensis (Lucena), Omalonyx unguis d'Orbigny e Pisidium sp. para a área Interserrana. Os moluscos sugerem, em linhas gerais, características palustres na base do Holoceno inicial. Durante o Holoceno médio, eles sugerem maior umidade (incremento no padrão de precipitações) $c a$. 4,9 ka AP e ca. 4,7 ka AP. No início do Holoceno final, os moluscos indicam una tendência árida interrompida por eventos úmidos curtos $c a$. 2,5 ka AP e $c a$. 2,2 ka AP. A integração e correlação dos registros de moluscos em uma escala espacial e temporal permitiu reconhecer deslocamentos geográficos (i. e. Retidiscus reticulatus Fonseca \& Thomé), os quais representam respostas bióticas às variações climáticas do Holoceno assim como diferenças ambientais locais em sequências estratigráficas de cronologias correlacionáveis.

Palavras-chaves: moluscos continentais, contextos de deposição, paleoambientes, Quaternário final, área Interserrana Bonaerense, Formação Luján. 


\section{INTRODUCCIÓN}

Los moluscos continentales han demostrado ser útiles como indicadores paleoambientales, tanto a nivel mundial (e.g. Castillo et al., 2002; Rousseau et al., 2001, 2006; Moine et al., 2008) como en la Argentina (Miquel \& Steffan, 2005; De Francesco \& Hassan, 2009; Aguirre et al., 2011; Miquel \& Aguirre, 2011). Sin embargo, en el sudeste de la Provincia de Buenos Aires sólo se han llevado a cabo estudios referidos a algunos sitios arqueológicos puntuales (e.g. Steffan 2007, Steffan et al., 2010; Bonomo et al., 2013) y no existe aún un análisis paleoambiental a nivel regional basado en el registro malacológico.

Desde el inicio de las investigaciones arqueológicas del área Interserrana Bonaerense (sudeste de la Provincia de Buenos Aires, "región Pampeana", Argentina) (Figura 1) ha existido un interés particular por reconocer las características de los parámetros físicos (ambiental) y biológico durante la ocupación de las poblaciones humanas (e.g. Politis, 1984; Martínez, 1999; Madrid et al., 2002). En el área de estudio existen numerosos estudios del paleoambiente basados en registros polínicos (entre otros, Prieto, 1996; Quattrocchio et al., 2008) y de vertebrados (Tonni et al., 1999, 2001) en contraste con la escasez de evidencias basadas en invertebrados y en moluscos en particular (Bonadonna et al., 1999; Zárate et al., 2000). En los sitios arqueológicos del sudeste de la Provincia de Buenos Aires la preservación de los microfósiles no siempre es óptima, por lo que surge la necesidad de explorar la potencialidad de información que ofrecen los moluscos para el estudio del paleoambiente.

En depósitos cuaternarios continentales del área Interserrana Bonaerense, la malacofauna es un componente biogénico común, abundante y de excelente preservación, particularmente en sitios arqueológicos ubicados entre la cuenca superior del arroyo Tapalqué y del río Quequén Salado (Figura 1). Los objetivos de este trabajo son: (i) caracterizar cuali y cuantitativamente los registros malacológicos - integrando los aspectos paleoecológicos, sistemáticos, tafonómicos y estadísticos - provenientes de sitios arqueológicos con dataciones radiocarbónicas disponibles (Holliday et al., 2003; Martínez, 2006; Massigoge, 2007; Messineo \& Politis, 2007); (ii) analizar las variaciones temporales y espaciales de los registros malacológicos locales; (iii) aportar información adicional independiente sobre los contextos arqueológicos tratados en relación con los paleoambientes continentales durante el Cuaternario tardío de la región Bonaerense, llenando un vacío de conocimiento en lo que se refiere a macroinvertebrados (moluscos), en contraste con una mayor cantidad de información disponible basada en otras fuentes de evidencia (entre otros, polen, Prieto, 1996; Quattrocchio et al., 2008; vertebrados, Tonni et al., 1999, 2001).

\section{MATERIAL Y MÉTODOS}

Los tres sectores geográficos analizados en este trabajo son: I) cuenca media del río Quequén Grande, II) sudoeste del área Interserrana y III) cuenca superior del arroyo Tapalqué.
En el sector I se analizaron tres sitios de la localidad arqueológica Paso Otero (PO1, PO3, PO5). En el II, se relevó el sitio Cortaderas 1 (COR) y dos perfiles ubicados en la margen izquierda de la cuenca inferior del río Quequén Salado, Perfil 1 (QS-P1) y 2 (QS-P2) (Tabla 1), ubicados a $10 \mathrm{~km}$ aguas arriba del sitio Quequén Salado 1 (QS1) (ver Figura 1). En cada sector seleccionado (I, II, III, Figura 1; Tablas 1,2) se muestrearon los perfiles estratigráficos expuestos luego de las tareas de excavación de los sitios arqueológicos y dos perfiles de la barranca izquierda del río Quequén Salado (Figura 1; Tabla 1). En cada columna se efectuaron muestreos sistemáticos, cada $10 \mathrm{~cm}$, del mismo peso $(c a .7 \mathrm{~kg})$ de sedimento de la Formación Luján (Fidalgo et al., 1973). El peso elegido obedece a que estudios previos sobre malacofauna cuaternaria continental mostraron que 2-2,5 $\mathrm{kg}$ representa el tamaño mínimo adecuado para efectuar un estudio taxonómico básico (Tonni et al., 2001; Aguirre et al., 2010), mientras en otras regiones geográficas $c a .10 \mathrm{~kg}$ de muestra resultaron estériles y otros autores no mencionan el volumen utilizable o contabilizan el esfuerzo realizado (horas por investigador) en la obtención del material. Se decidió seguir la metodología establecida por Steffan (2009) y modificada de Rousseau et al. (2001). El peso de la muestra se estableció con un criterio amplio ya que a priori se desconoce la proporción de sedimentos de los diferentes perfiles analizados. Complementariamente, se incluyó el material malacológico recuperado del cernidor de la excavación arqueológica, sitio Campo Laborde (CL) y Calera (CA). Asimismo, se efectuaron recolecciones de malacofauna moderna en los alrededores de cada uno de los sitios arqueológicos. Para ello, se siguieron las recomendaciones de Naranjo García \& Gómez Espinosa (2004) adecuadas al tipo de ambiente bajo estudio. Se muestreó desde la superficie del agua hasta $15 \mathrm{~cm}$ profundidad y la planicie de inundación de los cursos de agua analizados. Esta metodología provee datos para conocer la malacofauna viviente en el lugar y observaciones sobre su hábitat y hábito (modo de vida).

En el laboratorio, las muestras fueron lavadas bajo agua corriente utilizando tamices de abertura de malla de $0,5 \mathrm{~mm}$ para recuperar las conchas de moluscos. Los ejemplares ilustrados fueron fotografiados en el Servicio de Microscopía Electrónica de Barrido del Museo Argentino de Ciencias Naturales "Bernardino Rivadavia" (modelo Philips XL 30 TMP) y/o con lupa binocular Nikon ZMZ1000 con cámara Nikon Coolpix S10 adosada y/o con cámara Nikon D3100. Previamente el material seleccionado de cada taxón, de excelente preservación, fue limpiado manualmente y/o mediante limpiador ultrasónico (Clean 01 y Lilis 3.8) a intervalos de 5 segundos según el tamaño y fragilidad de las conchillas. El estado de preservación de las conchillas se evaluó a partir de la observación de los atributos tafonómicos de los taxones más representativos en todas las muestras (Heleobia parchappii y Succinea meridionalis), ya que no todos los taxones están presentes en los sitios, condición que impide considerar la totalidad de la malacofauna para establecer comparaciones ambientales significativas entre los distintos sitios. En el análisis de las modificaciones 


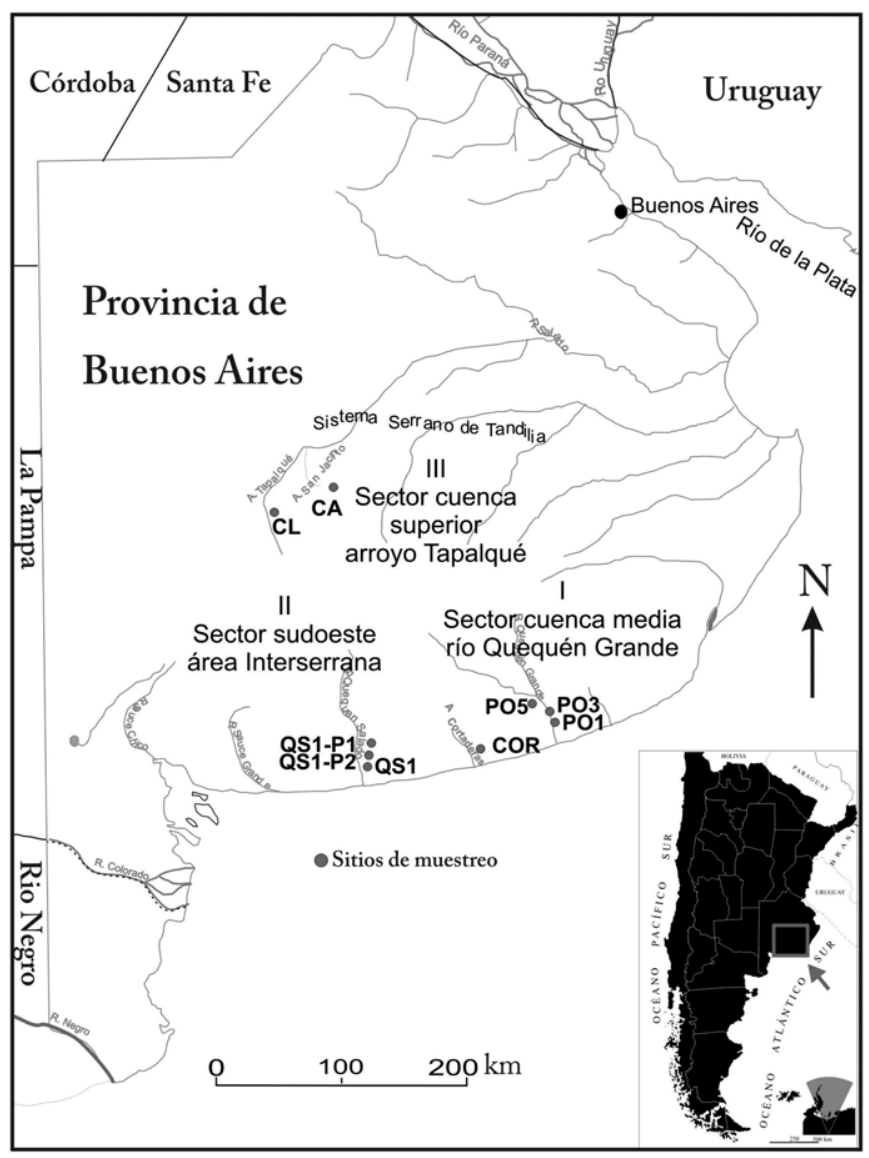

Figura 1. Ubicación de los perfiles de muestreo en el área de estudio (Área Interserrana Bonaerense, región Pampena, Argentina). Ubicación de la provincia de Buenos Aires y provincias aledañas (Córdoba, Santa Fe, La Pampa, Río Negro). Se muestran perfiles de sitios arqueológicos analizados.

Figure 1. Locations of sampling profiles in the studied area (Interserrana Bonaerensean area, Pampean region, Argentine). Location of Buenos Aires province and bordering provinces (Córdoba, Santa Fe, La Pampa, Río Negro). The profiles of the archaeological sites analyzed are shown.

Tabla 1. Sistema de coordenadas Universal transversal de Mercator (UTM) de los puntos de muestreos analizados.

Table 1. Coordinate system Universal Transverse Mercator (UTM) of sampling points analyzed.

\begin{tabular}{|c|c|c|c|}
\hline Sectores del área de estudio & $\begin{array}{c}\text { Sitio arqueológico } \\
\text { (sigla) }\end{array}$ & $\begin{array}{c}\text { Perfiles de muestreo } \\
\text { (sigla) }\end{array}$ & Sistema de coordenadas UTM (m.) \\
\hline \multirow{6}{*}{$\begin{array}{l}\text { I. Cuenca media del río Quequén } \\
\text { Grande }\end{array}$} & Paso Otero 1 & & \multirow{2}{*}{21 H 3637655715349} \\
\hline & PO 1 & & \\
\hline & Paso Otero 3 & & \multirow{2}{*}{21 H 3152885768935} \\
\hline & PO 3 & & \\
\hline & Paso Otero 5 & & \multirow{2}{*}{21 H 3147125769631} \\
\hline & PO 5 & & \\
\hline \multirow{5}{*}{ II. Sudoeste del área Interserrana } & Cortaderas 1 & & \multirow{2}{*}{21 H 2671945755231} \\
\hline & $\mathrm{COR}$ & & \\
\hline & & Quequén Salado & \multirow{2}{*}{20 H 7114945709449} \\
\hline & & QS-P 1 & \\
\hline & & QS-P 2 & $20 \mathrm{H} 7113035709516$ \\
\hline \multirow{4}{*}{$\begin{array}{l}\text { III. Cuenca superior del arroyo } \\
\text { Tapalqué }\end{array}$} & Campo Laborde 1 & & \multirow{2}{*}{$20 \mathrm{H} 7327175901837$} \\
\hline & $\mathrm{CL}$ & & \\
\hline & Calera & & \multirow{2}{*}{20 H 7456585903539} \\
\hline & $\mathrm{CA}$ & & \\
\hline
\end{tabular}


tafonómicas de los especímenes se consideró la fragmentación para discriminar entre condiciones de depositación en un ambiente lacustre o fluvial (menor grado de fragmentación) con corrientes y de mayor energía (mayor grado de fragmentación). Asimismo, se observó la corrasión (abrasión/ disolución, producidos por una combinación de factores físicos, químicos, o ambos y no discriminables) (Kotzian \& Simões, 2006). Otras modificaciones observadas en las conchillas son la incrustación interna y la recristalización (depositaciones químicas, e.g. carbonato de calcio), ya que aportan información para caracterizar las condiciones postentierro, y la naturaleza de los depósitos. La precipitación de carbonato de calcio es típica de algunos restos arqueológicos recuperados en depósitos donde la humedad es insuficiente (e.g. Lyman, 1994).

El análisis cuantitativo efectuado consistió en el cálculo de la abundancia relativa de individuos por especie, por nivel estratigráfico y por localidad/sitio. Se contabilizaron las conchillas enteras y, en caso de las fragmentadas, dependiendo de las especies, se consideró un individuo a la fracción que permitiera la identificación inequívoca de un ejemplar (i.e., última vuelta en Heleobia, ombligo en conchillas planospirales como Biomphalaria, Drepanotrema, Antillorbis). Se aplicaron los índices de riqueza de Margalef (d) y de diversidad específica de Shannon-Wiener (H”). Una estimación de similitud taxonómica se efectuó aplicando el Coeficiente de Asociación de Jaccard -CAJ- (Cheetham \& Hazel, 1969) ya que el mismo no considera las ausencias compartidas, situación frecuente en las matrices de datos (muestras de cada sitio por taxones presentes), tipo de sitios y área analizada. Con el objeto de visualizar las relaciones existentes (similitud taxonómica) entre los sitios arqueológicos se empleó la técnica de cluster analysis. Los valores oscilan entre 0 y 1, representando 1 la máxima similitud. Como medida de la distorsión que se produce sobre los datos de la matriz de similitud se calculó el Coeficiente de Correlación Cofenética (Rohlf, 1970). Para visualizar la distribución espacial entre los sitios se realizó un análisis de coordenadas principales (PCA, Principal Coordinate Analysis, Gower, 1966). Los análisis mencionados se realizaron utilizando el programa PAST v 2.16 (Hammer et al., 2001).

En este trabajo no se realizaron dataciones radiocarbónicas, por lo tanto las edades mencionadas fueron tomadas de trabajos publicados. Las edades fueron calibradas con el programa CALIB con la base de datos intcal13.14c (Reimer et al., 2013) (Tabla 2). Se efectuó una síntesis de la información disponible sobre requerimientos ecológicos y distribución de los taxones recuperados, a partir de datos propios, recopilaciones bibliográfica y comparaciones con material depositado en colecciones de instituciones nacionales y extranjeras: Museo de La Plata (MLP); Museo Argentino de Ciencias Naturales "Bernardino Rivadavia" (MACN), Muséum d'Histoire Naturelle de Paris (MNHN). La asignación de categorías taxonómicas a nivel supragenérico de gasterópodos está basada en Bouchet \& Rocroi (2005). Las interpretaciones paleoecológicas se basan en información bibliográfica sobre el biotopo de cada taxón (Gaillard \&
Castellanos, 1976; Cazzaniga, 1982; Bonetto et al., 1990; Rumi, 1991; Castellanos \& Miquel, 1991; Landoni, 1992; Ituarte, 1996; Aguirre \& Farinati, 2000; Aramayo et al., 2002; De Francesco \& Isla, 2004; Miquel \& Steffan, 2005; Simone, 2006; Gutiérrez Grégoric et al., 2006; Rumi et. al., 2007; De Francesco \& Hassan, 2009).

\section{AREA DE ESTUDIO: CLIMA MODERNO, SÍNTESIS GEOLÓGICA Y CRONOLOGÍA}

\section{Clima moderno}

El clima actual del área Interserrana Bonaerense $\left(37^{\circ} 00^{\prime} / 38^{\circ} 49^{\prime} \mathrm{S} ; 58^{\circ} 15^{\prime} / 60^{\circ} 32^{\prime} \mathrm{O}\right)$ es mesotérmico, caracterizado por temperaturas medias anuales de $13^{\circ}$ a $17^{\circ} \mathrm{C}$ y precipitaciones de $600 \mathrm{~mm}$ a $1.200 \mathrm{~mm}$ anuales. Desde el punto de vista fitogeográfico corresponde a la Provincia Pampeana del Domino Chaqueño (Cabrera \& Willink, 1973). Zoogeográficamente corresponde al Dominio Pampásico de la Subregión Guayano-Brasileña (Ringuelet, 1955, 1961). Los estudios paleoambientales efectuados en los perfiles estratigráficos de las cuencas analizadas reconocen que el clima del Holoceno se caracteriza por un período inestable, con cambios cortos en la temperatura y humedad/precipitación durante el Holoceno medio y tardío (Bonadonna et al., 1999; Zárate et al., 2000; Prado et al., 2005; Steffan, 2007; Grill et al., 2007; Osterrrieth et al., 2008; Gutiérrez et al., 2011). Específicamente durante el Holoceno medio se reconoce un episodio de máxima temperatura ("Máximo Termal del Holoceno medio", "Hypsithermal") reconocido tanto a nivel mundial como regional y local (Briner et al., 2006; Aguirre, 1993, 2002).

\section{Síntesis geológica}

En el sector analizado del área Interserrana se reconocieron sedimentos correspondientes al Miembro Río Salado de la Formación Luján. Allí se encuentran los tres sectores principales seleccionados para este estudio: I, Cuenca media del río Quequén Grande, II, Sudoeste del área Interserrana y III, Cuenca superior del arroyo Tapalqué. El área de estudio se encuentra entre los cordones Serranos de Tandilla y de Ventania (Figura 1). El Sistema Serrano de Tandilia, conocido también como Sierras Septentrionales, de la provincia de Buenos Aires (Fidalgo et al., 1986), constituye un cordón discontinuo (50 y 250 metros sobre el nivel del mar) de más de $300 \mathrm{~km}$ de largo interrumpido por varios valles y lomadas emergentes en la llanura. Presenta una orientación general noroeste-sudeste (Teruggi \& Kilmurray, 1980). El sistema Serrano de Ventania, también conocido como Sierras Australes, de la Provincia de Buenos Aires, se dispone como una cadena montañosa de $180 \mathrm{~km}$ de largo y $60 \mathrm{~km}$ de ancho ubicado en el sureste de la provincia. Presenta orientación noroeste-sureste. Su altura supera los $1.000 \mathrm{~m}$ sobre el nivel del mar (ver Dimieri et al., 2005).

En la estratigrafía de valles del área Interserrana, Fidalgo et al. (1973) incluyeron las unidades cronoestratigráficas conocidas como Lujanense y Platense (sensu Frenguelli, 1950) en la Formación Luján, conceptualizando y definiéndolos 
como unidades litoestratigráficas subordinadas (miembros): Miembro Guerrero (palustre) y Miembro Río Salado (fluvial). Las cronologías publicadas para la Formación Luján abarcan desde 28 ka AP y 2.3 ka AP (e.g. Figini et al., 1998; Tonni et al., 1999; Holliday et al., 2003). Entre ambos miembros (Guerrero y Río Salado) se identificaron las unidades pedoestratigráficas o paleosuelos, Puesto Callejón Viejo (Holoceno temprano) y Puesto Berrondo (Holoceno medio/ tardío) (Fidalgo et al., 1973; Zárate et al., 2000).

El paleosuelo Puesto Berrondo se desarrolla en la parte superior del Miembro Río Salado y se encuentra cubierto de sedimentos eólicos de la Formación La Postrera (mantos loésicos) (Fidalgo et al., 1973; Zárate et al., 2000). Culminan la secuencia sedimentaria los eventos de inundación más recientes que aportaron material aluvial para el desarrollo de suelos modernos (Zárate et al., 2000).

\section{Geoarqueología}

I. Cuenca media del río Quequén Grande. Los perfiles estratigráficos han sido estudiados previamente por diferentes investigadores desde el punto de vista estratigráfico, geoarqueológico y cronológico (e.g. Johnson et al., 1998; Holliday et al., 2003; Favier Dubois, 2006). La secuencia estratigráfica de la localidad Paso Otero ha sido sintetizada en tres unidades litoestratigráficas, aunque en cada uno de los sitios se registran facies fluviales a fluvio lacustres que aportan material parental para el desarrollo de suelos a escala local datados previamente entre $\sim 10,4$ y 2,3 ka AP (Figura 2, Tabla 2) (e.g. Johnson et al., 1998; Holliday et al., 2003; Martínez, 2006). En los sitios de la localidad arqueológica Paso Otero (PO1, PO3, PO5) Martínez $(1999,2006)$ y Kaufmann \& Gutiérrez (2004) entre otros, estudiaron tecnologías de subsistencia, dispersión de huesos de guanaco y aspectos vinculados con la funcionalidad de los sitios arqueológicos. Martínez $(1999,2006)$ propuso que en el sitio Paso Otero 5 se produjeron ocupaciones breves ligadas a la caza y/o carroñeo de megamamíferos durante el Holoceno temprano (Figura 2; Tabla 2).

II. Sudoeste del área Interserrana. En el sector de la cuenca inferior del río Quequén Salado la geología ha sido estudiada y correlacionada con distintas unidades litoestratigráficas definidas a escala regional (e.g. Mormeneo, 1992; Farinati \& Zavala, 1995). En dicho sector han sido reconocidas al menos cuatro unidades (Mormeneo, 1992), de la cuales en este trabajo se analizan de las unidades II, IIIa, b y IV. El techo de los perfiles analizados (Unidad IIb) han sido correlacionados con el desarrolló un suelo palustre (identificado en el sitio arqueológico Quequén Salado 1 -QS1- ( 960 - 360 años AP) (Figura 2; Tabla 2) (Steffan et al., 2010), donde Madrid et al. (2002) analizaron la arqueología microrregional (Figura 1). En el sitio Cortaderas 1 -COR-, ubicado en el arroyo homónimo, la secuencia estratigráfica se compone por dos unidades y entre ambas se desarrolló un paleosuelo parcialmente

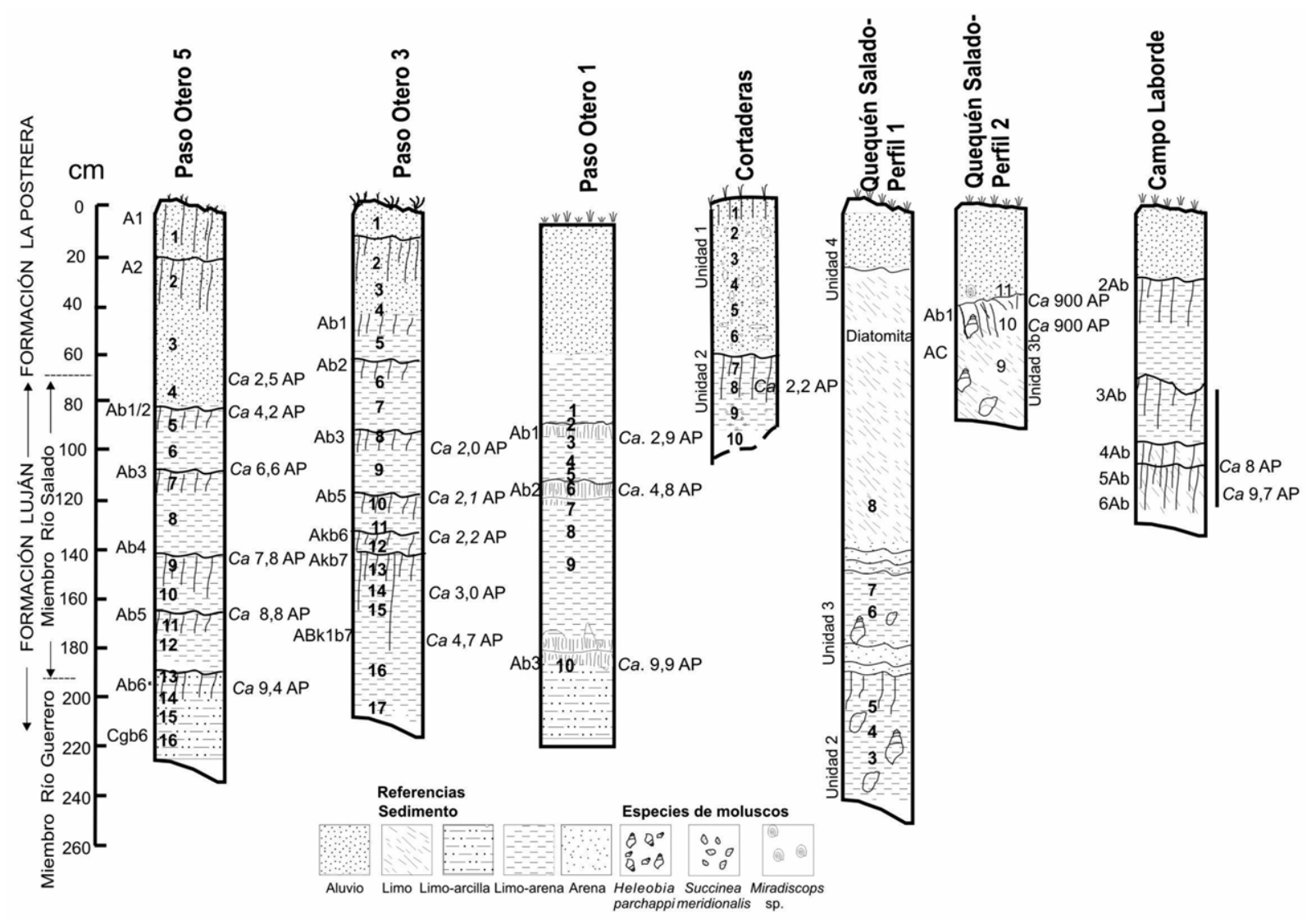

Figura 2. Síntesis de la sucesión sedimentaria de los perfiles estratigráficos analizados.

Figure 2. Synthesis of the sedimentary succession of the stratigraphical profiles analyzed. 
Tabla 2. Síntesis de la cronología de los sitios analizados. Todas las edades se presentan calibradas. Tipo de muestra datada: (a) Materia orgánica de suelo $\left(^{b}\right)$ Lama guanicoe, $\left({ }^{c}\right)$ Megafauna. *Indica edades con escaso contenido de colágeno. (Referencias completas en Johnson et al., 1998; Madrid et al., 2002; Politis et al., 2005; Martínez, 2006; Messineo \& Politis, 2007; Masigoge, 2007).

Table 2. Synthesis of the chronology of the sites analyzed. All ages are calibrated. Type of sample dated: $\left({ }^{(a)}\right)$ soil organic matter, $\left({ }^{b}\right)$ Lama guanicoe, $\left({ }^{c}\right)$ Megafauna. *Indicate ages with low collagen content. (Complete references in Johnson et al., 1998; Madrid et al., 2002; Politis et al., 2005; Martínez, 2006; Messineo \& Politis, 2007; Masigoge, 2007).

\begin{tabular}{|c|c|c|c|c|c|}
\hline Sector & Sitio & Unidad & Edad ${ }^{14} \mathrm{C}$ (años AP) & $\begin{array}{c}\text { Edad calibrada } \pm 2 \text { sigma } \\
\text { (probabilidad) }\end{array}$ & Referencia \\
\hline \multirow{12}{*}{ 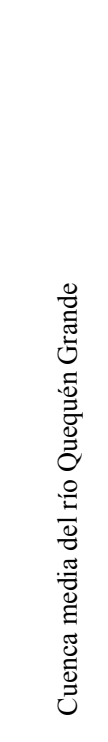 } & \multirow{4}{*}{ PO 1} & $\mathrm{Ab} 1$ & $2.974 \pm 48^{\mathrm{a}}$ & AC 1.057: AC $827(0,99 \%)$ & Johnson et al., 1998 \\
\hline & & $\mathrm{Ab} 2$ & $3.056 \pm 42^{\mathrm{b}}$ & AC 1.421: AC $1.210(1 \%)$ & Martínez, 2006 \\
\hline & & & $4.855 \pm 105^{\mathrm{a}}$ & AC 3.812: AC $3.489(0,83 \%)$ & Johnson et al., 1998 \\
\hline & & Ab3 & $9.950 \pm 65^{\mathrm{a}}$ & AC 9.692: AC $9.283(0,97 \%)$ & Johnson et al., 1998 \\
\hline & \multirow{6}{*}{ PO3 } & $\mathrm{Ab} 2$ & $1.571 \pm 64^{\mathrm{a}}$ & DC 377: DC 617 (0,98\%) & Martínez, 2006 \\
\hline & & Ab3 & $2.061 \pm 70^{\mathrm{a}}$ & AC 212: DC $79(0,94 \%)$ & Martínez, 2006 \\
\hline & & Ab5 & $2.137 \pm 67^{\mathrm{a}}$ & AC 369: AC $36(0,98 \%)$ & Martínez, 2006 \\
\hline & & Akb6 & $2.402 \pm 81^{\mathrm{a}}$ & AC 784: AC 364 (1\%) & Martínez, 2006 \\
\hline & & Akb7 & $3.003 \pm 63^{\mathrm{a}}$ & AC 1.411: AC $1.050(1 \%)$ & Martínez, 2006 \\
\hline & & $\mathrm{ABk} 1 \mathrm{~b} 7$ & $4.713 \pm 63^{\mathrm{a}}$ & AC 3.635: AC $3.483(0,57 \%)$ & Martínez, 2006 \\
\hline & \multirow{6}{*}{ PO5 } & Bk1 & $2.490 \pm 70^{\mathrm{a}}$ & AC 790: AC $428(0,99 \%)$ & Martínez, 2006 \\
\hline & & $\mathrm{Ab} 1 / \mathrm{Ab} 2$ & $4.210 \pm 65^{\mathrm{a}}$ & AC 2.918: AC $2.617(0,98 \%)$ & Martínez, 2006 \\
\hline \multirow{8}{*}{ 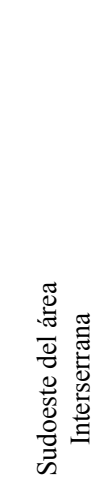 } & & Ab3 & $6.629 \pm 129^{\mathrm{a}}$ & AC 5.759: AC 5.320 (1\%) & Martínez, 2006 \\
\hline & & $\mathrm{Ab} 4$ & $7.794 \pm 71^{\mathrm{a}}$ & AC 6.829: AC $6.462(0,98 \%)$ & Martínez, 2006 \\
\hline & & Ab5 & $8.793 \pm 89^{\mathrm{a}}$ & AC 8.021: AC $7.611(0,75 \%)$ & Martínez, 2006 \\
\hline & & Ab6 & $9.399 \pm 116^{\mathrm{a}}$ & AC 8.939: AC $8.329(0,86 \%)$ & Martínez, 2006 \\
\hline & COR & $\mathrm{A} 1$ & $2.270 \pm 190^{\mathrm{b}}$ & AC 803: DC $81(1 \%)$ & Massigoge, 2007 \\
\hline & \multirow{3}{*}{ Q S 1} & $\mathrm{Ab} 1$ & $360 \pm 40^{\mathrm{b}}$ & DC 1.537: DC $1.636(0,52 \%)$ & Madrid et al., 2002 \\
\hline & & $\mathrm{AC}$ & $940 \pm 40^{\mathrm{b}}$ & DC 1.019: DC $1.183(1 \%)$ & Madrid et al., 2002 \\
\hline & & $\mathrm{AC}$ & $960 \pm 40^{b}$ & DC 1.012: DC $1.164(0,99 \%)$ & Madrid et al., 2002 \\
\hline \multirow{12}{*}{ 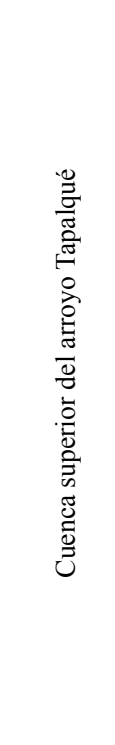 } & \multirow{6}{*}{$\mathrm{CL}$} & $5 \mathrm{Ab}$ & $7.750 \pm 250^{c}$ & AC 7.196: AC $6.091(0,98 \%)$ & Messineo \& Politis, 2007 \\
\hline & & $5 \mathrm{Ab}$ & $7.960 \pm 100^{\mathrm{a}}$ & AC 7.084: AC $6.600(0,99 \%)$ & Messineo \& Politis, 2007 \\
\hline & & $6 \mathrm{Ab}$ & $8.080 \pm 200^{c}$ & AC 7.524: AC 6.587 (0,99\%) & Messineo \& Politis, 2007 \\
\hline & & $6 \mathrm{Ab}$ & $8.090 \pm 190^{\mathrm{a}}$ & AC 7.517: AC $6.600(1 \%)$ & Messineo \& Politis, 2007 \\
\hline & & $6 \mathrm{Ab}$ & $8.720 \pm 190^{c}$ & AC 8.293: AC $7.453(0,99 \%)$ & Messineo \& Politis, 2007 \\
\hline & & $6 \mathrm{Ab}$ & $9.730 \pm 290^{c}$ & AC 10.110: AC $8.322(1 \%)$ & Messineo \& Politis, 2007 \\
\hline & \multirow{6}{*}{$\mathrm{CA}$} & Cubeta & $1.748 \pm 42^{\mathrm{b}}$ & DC 209: DC $394(0,95 \%)$ & Politis et al., 2005 \\
\hline & & Cubeta & $2.075 \pm 44^{\mathrm{b}}$ & AC 200: DC $21(1 \%)$ & Politis et al., 2005 \\
\hline & & Cubeta & $2.232 \pm 55^{\mathrm{b}}$ & AC 398: AC 175 (1\%) & Politis et al., 2005 \\
\hline & & Cubeta & $3.005 \pm 66^{\mathrm{b}}$ & AC 1.413: AC $1.050(1 \%)$ & Politis et al., 2005 \\
\hline & & Cubeta & $3.008 \pm 44^{\mathrm{b}}$ & AC 1.329: AC $1.117(0,83 \%)$ & Politis et al., 2005 \\
\hline & & Cubeta & $3.390 \pm 170^{\mathrm{b}}$ & AC 2.141: AC $1.282(0,99 \%)$ & Politis et al., 2005 \\
\hline
\end{tabular}


erosionado (Favier Dubois, en Massigoge, 2007). En este sitio, se estudiaron aspectos corológicos de la mastofauna y de la ocupación humana vinculadas a la talla del material lítico, consumo de guanaco y venado durante el Holoceno tardío (Tabla 2) (Politis et al., 1983; Massigoge, 2007).

III. Cuenca superior del arroyo Tapalqué. La cuenca superior del arroyo Tapalqué se ubica en el norte del área Interserrana y en el extremo noroccidental del sistema serrano de Tandilia (Figura 1). En Campo Laborde -CL-, se reconocen sedimentos asignables a los dos miembros de la Formación Luján. Entre ambos se desarrollan horizontes de paleosuelo ( 9,7 - 8 ka AP) (Figura 2, Tabla 2) (Messineo $\&$ Politis, 2007). El sitio arqueológico representa un lugar de caza/carroñeo de Megatherium americanun a orillas de un pantano y cuyas evidencias de megafauna pleistocena, sobreviviente en el Holoceno temprano, fue tratada por Politis \& Messineo (2008). En el sector del arroyo Tapalqué se estudió el sitio Calera -CA-, próximo a las nacientes del arroyo San Jacinto, donde se han identificado cubetas excavadas en sedimentos correspondientes a los miembros Guerrero y Río Salado, constituyendo un rasgo transgresivo entre $\sim 3,4$ ka AP y 2 ka AP (Steffan 2009) (Tabla 2). En el sitio Calera Politis et al. (2005) reconocieron cubetas excavadas en el sedimento vinculadas a prácticas rituales múltiples y depósito ceremonial de objetos.

\section{RESULTADOS}

\section{Composición taxonómica de la malacofauna en los sitios}

La totalidad de la malacofauna recuperada está representada por 11 familias de gasterópodos continentales (Cochliopidae, Chilinidae, Lymnaeidae, Physidae, Planorbidae, Ancylidae, Helicodiscidae, Charopidae, Punctidae, Scolodontidae y Succineidae), y una de bivalvos (Sphaeridae), con 14 géneros de gasterópodos (Heleobia, Chilina, Lymnaea, "Aplexa", Drepanotrema, Biomphalaria, Antillorbis, Uncancylus, Zilchogyra, Retidiscus, Paralaoma, Miradiscops, Succinea y Omalonyx) y un género de bivalvo (Pisidium) (Figuras 3, 4; Tabla 3). Adicionalmente, se registró una especie introducida, Paralaoma servilis (Shuttleworth, 1852), de origen incierto (Tablas 3, 4) (ver Miquel et al., 2007).

Los resultados preliminares de las recolecciones realizadas en las áreas de muestreo permiten reconocer la presencia de algunos taxones de moluscos modernos que han sido identificados en los sitios arqueológicos (es decir, Heleobia parchappii, Biomphalaria peregrina, Succinea meridionalis; Tablas 3, 4). En la cuenca media del río Quequén Grande se identificaron cuatro especies de gasterópodos de distintos ambientes: fluvio-lacustres (H. parchappii, Chilina parchappii, B. peregrina) e higrófilo o de sectores pantanosos (S. meridionalis) (Tablas 3, 4). En el río Quequén Grande se recuperaron $H$. parchappii y $B$. peregrina. En las recolecciones del arroyo San Jacinto (área Interserrana, Figura 1) y del arroyo Tapalqué se identificaron cinco taxones de gasterópodos (H. parchappii, Ch. parchappii, B. peregrina, U. concentricus, Miradiscops sp.) y un bivalvo (Pisidium sp.).
Clase GASTROPODA Cuvier, 1797

Orden SORBEOCONCHA Ponder \& Lindberg, 1997

Superfamilia RISSOOIDEA Gray, 1847

Familia COCHLIOPIDAE Tryon, 1866

Heleobia Stimpson, 1865

Heleobia parchappii (d'Orbigny, 1835)

(Figuras 3Aa,b; Tablas 3,4)

1835 Paludina parchappii d'Orbigny, p. 30.

1840 Palusdestrina parchappii d'Orbigny, p. 383, pl. 48, figs. 1-3.

1992 Heleobia parchappii Hershler \& Thompson, p. 55.

2006 Heleobia parchappii Gutiérrez Grégoric et al., p. 53, fig. 7.

Materiales recuperados. FCS-IN RQG 9. Paso Otero 1, Holoceno medio, 4.870 ejemplares (ejs.); FCS-IN RQG 52. Paso Otero 1, Holoceno temprano, 12 ejs.; FCS-IN RQG 66. Paso Otero 5, Holoceno medio, 1 ej.; FCS-IN RQG 79. Paso Otero 5, Holoceno temprano, 16 ejs.; FCS-IN RQG 121. Paso Otero 3, Holoceno tardío, 907 ejs.; FCS-IN C LA 231. Campo Laborde 1, Holoceno temprano, 5 ejs.; FCS-IN CAL 252. Calera, Holoceno tardío, 47 ejs.; FCS-IN ACOR 347. Arroyo Cortaderas, Holoceno tardío, 813 ejs.

Materiales comparados. 1 Lectotipo (NHM 1854.12.4.341). MLP 12440. Buenos Aires, río Santiago, arroyo Doña Flora; MLP 677. Balcarce, arroyo de Lobería, Buenos Aires; MLP 4015-1. Entre Ríos, arroyo Caribello, Colón.

Dimensiones de los materiales fotografiados. Alturas: 3,4 $\mathrm{mm}$ y $4,1 \mathrm{~mm}$.

Distribución actual en Argentina. Santa Fe, Entre Ríos, Buenos Aires, La Pampa y Río Negro en Argentina. Ambientes lénticos y lóticos.

Distribución estratigráfica. Pleistoceno-Actualidad.

Registros en el Cuaternario de Argentina. Pleistoceno, Formación Querandina Superior (Pre-Belgranense) de la cuenca bonaerense (Doering, 1884, p. 473). Holoceno, Buenos Aires, Laguna La Salada Grande (Aguirre, 1990), río Luján (Prieto et al., 2004), Bahía Blanca (Farinati \& Zavala, 1995; Aramayo et al., 2002), Formación Luján Miembro Guerrero y Miembro Río Salado de Paso Otero, Salto de Piedra y río Quequén Salado (Bonadonna et al., 1999; Figini et al., 1998); Mendoza: cuenca del río Atuel (De Francesco \& Dieguez, 2006).

Orden PULMONATA Cuvier en Blainville, 1814 Suborden BASOMMATOPHORA Schmidt, 1855 Familia LYMNAEIDAE Rafinisque, 1815

Lymnaea Lamarck, 1799

Lymnaea viator d'Orbigny, 1835

(Figuras 3Ca,b; Tablas 3,4) 


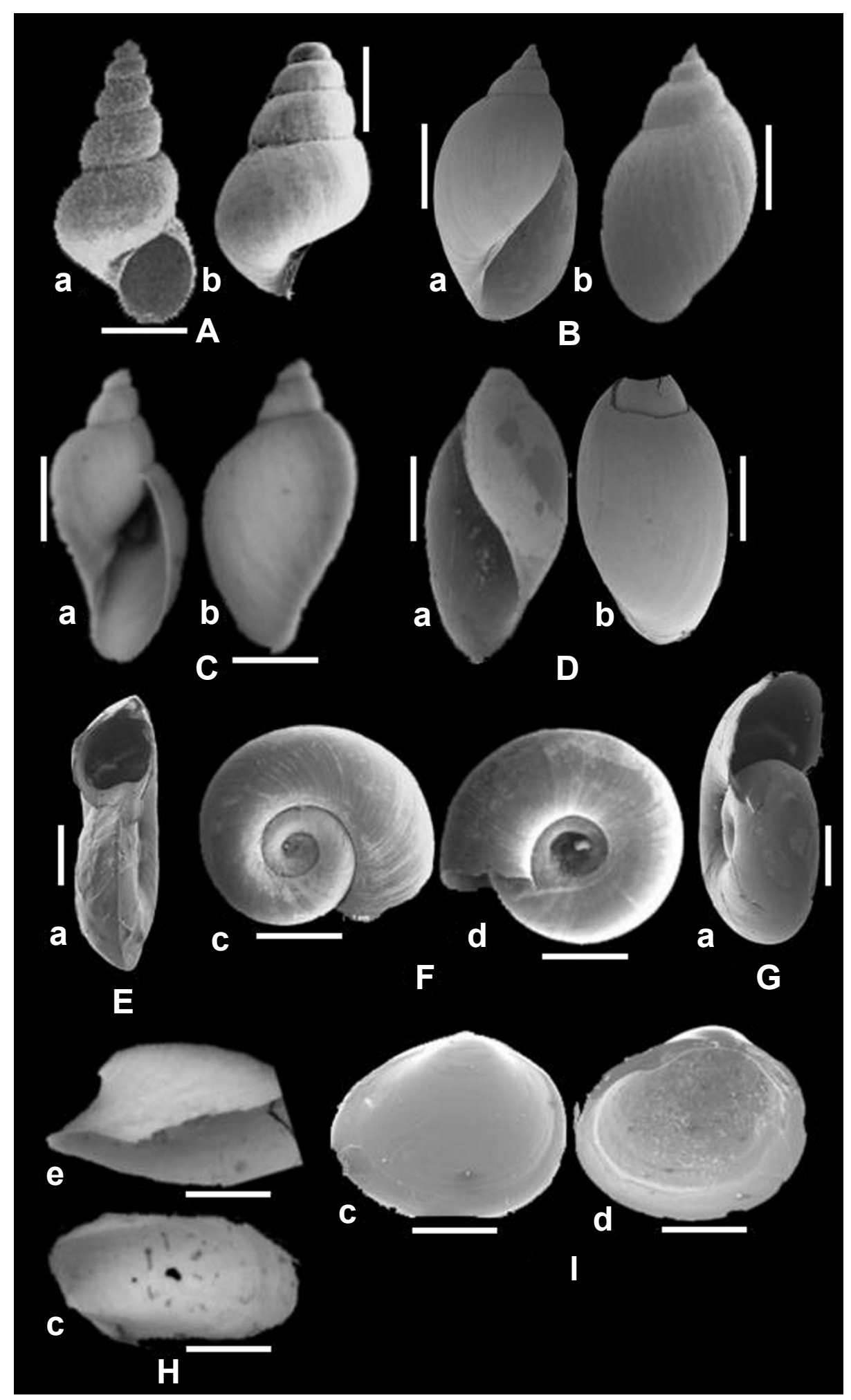

Figura 3. Moluscos acuáticos. Gastropodos: A, Heleobia parchappii, Paso Otero 1, Holoceno; B, Chilina parchappii, Paso Otero 1, Holoceno; C, Lymnaea viator, Cortaderas, Holoceno; D, "Aplexa” minor, Calera, Holoceno tardío; E, Drepanotrema kermatoides, Calera, Holoceno tardío; F, Biomphalaria peregrina, Paso Otero 1, Holoceno; G, Antillorbis nordestensis, Calera, Holoceno tardío; $\mathbf{H}$, Uncancylus concentricus, Calera, Holoceno tardío. Bivalvo: I, Pisidium sp., Calera, Holoceno tardío. Abreviaturas: a, vista apertural; b, vista adapertural; c, vista dorsal; d, vista umbilical; e, lateral. Escalas: $A, C, F=1 \mathrm{~mm} ; B, D, E=2 \mathrm{~mm} ; E, F=200 \mu \mathrm{m} ; \mathrm{I}=500 \mu \mathrm{m}$.

Figure 3. Aquactic mollusks. Gastropods: A, Heleobia parchappii, Paso Otero 1, Holocene; B, Chilina parchappii, Paso Otero 1, Holocene; C, Lymnaea viator, Cortaderas, Holocene; D, "Aplexa" minor, Calera, late Holocene; E, Drepanotrema kermatoides, Calera, late Holocene; F, Biomphalaria peregrina, Paso Otero 1, Holocene; G, Antillorbis nordestensis, Calera, late Holocene; $\mathbf{H}$, Uncancylus concentricus, Calera, late Holocene. Bivalve: I, Pisidium sp., Calera, late Holocene. Abbreviations: a, apertural view; b, adapertural view; c, dorsal view; d, umbilical view; e, lateral view. Scale bars: $A, C, F=1 \mathrm{~mm} ; B, D, E=2 \mathrm{~mm} ; E, F=200 \mu \mathrm{m} ; \mathrm{I}=500 \mu \mathrm{m}$. 
Tabla 3. Malacofauna terrestre y dulciacuícola recuperada en los sitios arqueológicos muestreados en el área Interserrana y viviente en áreas aledañas. Requerimientos ecológicos de los representantes modernos de los taxones estudiados.

Table 3. Terrestrial and freshwater malacofauna recovered in archaeological sites sampled in the Interserrana area and living in surrounding areas. Ecological requirements of the modern representatives of the taxa studied.

Sitios analizados del área

Interserrana bonaerense

$\begin{array}{llllllll}\text { PO } 1 & \text { PO } 3 & \text { PO } 5 & \text { COR } & \text { Q S } & \text { CL } & \text { CA } & \text { Vivientes }\end{array}$

Taxones identificados en el área

Clase GASTROPODA

Heleobia parchappii d'Orbigny, 1835

Chilina parchappii (d'Orbigny, 1835)

Lymnaea viator d'Orbigny, 1835

“Aplexa” minor (d’Orbigny, 1837)

Drepanotrema kermatoides (d'Orbigny, 1835)

Biomphalaria peregrina (d'Orbigny, 1835)

Antillorbis nordestensis (Lucena, 1954)

Uncancylus concentricus (d'Orbigny, 1835)

Zilchogyra costellata (d'Orbigny, 1835)

Retidiscus reticulatus Fonseca \& Thomé, 1995

Paralaoma servilis Shuttleworth, 1852

Miradiscops brasiliensis (Thiele, 1927)

Succinea meridionalis d'Orbigny, 1846

Omalonyx unguis (d'Orbigny, 1835)

Clase BIVALVIA

Pisidium sp.
Cerca de la superficie a poca profundidad, adherida a algas, vegetación sumergida, rocas, aguas salobres y dulces

Cerca de la superficie o a poca profundidad, adherida a algas

Fondos limosos, se entierra en épocas inhóspitas, asociada a macrófitas, ambientes de baja energía

Adherido a piedras, plantas acuáticas en la superficie

Sobre vegetación acuática, macrófitas

Sobre vegetación acuática, macrófitas, ambientes de baja energía

Sobre vegetación acuática

Sujeta a sustratos duros, hojas flotantes y tallos. Sumergida, adherida a la superficie inferior de la carpeta vegetal, euríoica

Terrestre o semiarbóreos, bajo piedras, troncos, ramas caídas, en descomposición. A orillas de lagunas. Cava unos centímetros

Terrestre, sustrato húmedo Terrestre, sustrato húmedo

Terrestre, bajo vegetales en descomposición, troncos, hojas muertas, humus

Higrófilia, suelos muy húmedos y acumulaciones de aguas pluviales

Higrófilia, sobre carpeta vegetal

Infaunal
1835 Limnoeus viator d'Orbigny, p. 24.

1837 Limneus viator d'Orbigny, p. 340, pl. 43, figs. 4-5. 1995 Lymnaea viator Castellanos \& Landoni, p. 777.

Materiales recuperados. FCS-IN ACOR 348. Arroyo Cortaderas; Holoceno tardío, 2 ejs.; FCS-IN ACOR 354. Arroyo Cortaderas; Holoceno tardío, 1 ej.; FCS-IN ACOR 360. Arroyo Cortaderas, Holoceno tardío, 2 ejs.

Materiales comparados. 1 Sintipo (NHM s/n). MACN-In 9.938. Buenos Aires: Punta Lara; MACN-In 11.836. Buenos Aires: Atalaya, Magdalena, Río de la Plata; MACN-In 36.848.
Mendoza: arroyo La Estacada, río Tunuyán, valle de Uco.

Dimensiones de los materiales fotografiados. Alturas: 3 $\mathrm{mm}$ y $4 \mathrm{~mm}$.

Distribución actual en Argentina. Desde las provincias de Río Negro y Buenos Aires, alcanzando el norte de Argentina a través de una faja por el centro-oeste del país. Vive en variedad de ambientes lénticos y lóticos (inclusive en aguas termales), asociada a macrófitas acuáticas.

Distribución estratigráfica. Pleistoceno-Actualidad.

Registros en el Cuaternario de Argentina. Pleistoceno, Mendoza, cuenca del río Atuel (De Francesco \& Dieguez, 
Tabla 4. Distribución (estratigráfica y geográfica moderna) y ambiente de los taxones identificados en el presente trabajo. Abreviaturas: ${ }^{*}=$ primer registro para el Holoceno; $\mathbf{X}=$ primer registro para la Argentina (Miquel \& Steffan, 2005); PL = Pleistoceno; $\mathbf{H}=\mathrm{Holoceno}$. Regiones de distribución geográfica moderna: Norte (Sureste de Brasil, Noroeste argentino, Chaqueña y Mesopotamia); Centro (Cuyo, Sierras Pampeanas); y Sur (norte y sur de Patagonia). (Basado en d'Orbigny, 1835; Landoni, 1992; Castellanos \& Miquel, 1991; Fonseca \& Thomé, 1995; Miquel \& Steffan, 2005; Simone, 2006).

Table 4. Distribution (modern stratigraphic and geographic) and environment of the taxa identified in this paper. Abbreviations: * First record by the Holocene; $\mathbf{X}=$ first record by Argentina (Miquel \& Steffan, 2005); PL = Pleistocene; $\mathbf{H}=$ Holocene. Modern geographical regions: North (Southeast of Brazil, Argentine Northwest, Chaco and Mesopotamia); Center (Cuyo, Pampean Ranges); and South (Northern and Southern Patagonia). (Based on d’Orbigny, 1835; Landoni, 1992; Castellanos \& Miquel, 1991; Fonseca \& Thomas, 1995; Miquel \& Steffan, 2005; Simone, 2006).

\begin{tabular}{|c|c|c|c|c|c|c|c|}
\hline \multirow[t]{2}{*}{ Taxón } & \multirow[t]{2}{*}{ Ambiente y Distribución } & \multirow[t]{2}{*}{ Ambiente } & \multicolumn{2}{|c|}{ 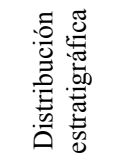 } & \multicolumn{3}{|c|}{ 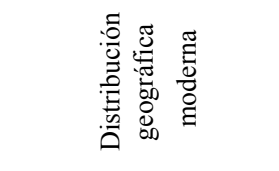 } \\
\hline & & & PL & $\mathrm{H}$ & Norte & Centro & Sur \\
\hline Heleobia parchappii d'Orbigny, 1835 & & Acuático & & & & & \\
\hline Chilina parchappii (d'Orbigny, 1835) & & Acuático & & & & & \\
\hline Lymnaea viator d'Orbigny 1835 & & Acuático & & & & & \\
\hline “Aplexa” minor (d'Orbigny, 1837) & & Acuático & & $\mathrm{X}$ & & & \\
\hline Drepanotrema kermatoides (d'Orbigny, 1835) & & Acuático & & $\mathrm{X}$ & & & \\
\hline Biomphalaria peregrina (d'Orbigny, 1835) & & Acuático & & & & & \\
\hline Antillorbis nordestensis (Lucena, 1954) & & Acuático & & $\mathrm{X}$ & & & \\
\hline Uncancylus concentricus (d’Orbigny, 1835) & & Acuático & & $\mathrm{X}$ & & & \\
\hline Zilchogyra costellata (d'Orbigny, 1835) & & Terrestre & & & & & \\
\hline Retidiscus reticulatus Fonseca \& Thomé, 1995 & & Terrestre & & $*$ & & & \\
\hline Paralaoma servilis (Shuttleworth, 1852) & & Terrestre & & & & & \\
\hline Miradiscops brasiliensis (Thiele, 1927) & & Terrestre & & & & & \\
\hline Succinea meridionalis d'Orbigny, 1846 & & Hidrófilo & & & & & \\
\hline Omalonyx unguis (d’Orbigny, 1835) & & Hidrófilo & & $\mathrm{X}$ & & & \\
\hline Pisidium sp. & & Acuático & & $\mathrm{X}$ & & & \\
\hline
\end{tabular}

2006; De Francesco et al., 2007). Holoceno, Calera (Buenos Aires) (este trabajo).

Familia CHILINIDAE Dall, 1870

Chilina Gray, 1828

Chilina parchappii (d'Orbigny, 1835)

(Figuras 3Ba,b; Tablas 3,4)

1835 Limnceus parchappii d'Orbigny, p. 24, pl. 25, fig. 4. 1837 Chilina parchappii d'Orbigny, p. 338, pl. 43, figs. 4-5. 1995 Chilina parchappii d'Orbigny. Castellanos \& Landoni, p. 777 , fig. 42 .

Materiales recuperados. FCS-IN RQG 1.1 Paso Otero 1; Holoceno medio, 2 ejs.; FCS-IN RQG 31; Paso Otero 1, Holoceno medio, 6 ejs.; FCS-IN RQG 37. Paso Otero 1; Holoceno medio), 1 ej.; FCS-IN RQG 110. Paso Otero 3, Holoceno tardío, 1 ej.

Materiales comparados. 7 Sintipos (NHM 1854.12.4.341). MLP 2287. Buenos Aires: Luján; MLP 29445. Buenos Aires: río Quequén Salado.

Dimensiones del material fotografiado. Altura: $11 \mathrm{~mm}$.

Distribución actual en Argentina. Litoral de Buenos Aires y
Patagonia norte, con relictos en limnótopos de aguas salobres en el interior del país (Mendoza, Salta).

Distribución estratigráfica. Pleistoceno-Actualidad.

Registros en el Cuaternario de Argentina. Pleistoceno: Buenos Aires: Miembro Guerrero; Formación Buenos Aires (Edad Bonaerense) (Necochea) (Castellanos \& Miquel, 1991; Figini et al., 1998). Holoceno: Miembro Río Salado en Salto de Piedra (Figini et al., 1998), Paso Otero 1 (Steffan, 2007).

Familia PHYSIDAE Fitzinger, 1833

Aplexa Fleming, 1820

"Aplexa" minor (d'Orbigny, 1837)

(Figuras 3Da,b; Tablas 3,4)

1837 Physa rivalis var. minor d'Orbigny, p. 341-342.

1995 Stenophysa marmorata Castellanos \& Landoni, p. 776, figs. 43a.

2004 “Aplexa” minor Scarabino, p. 351.

Materiales recuperados. FCS-IN CAL 256 (Calera cuadrícula 2, Holoceno tardío), 146 ejs.; FCS-IN CAL 265 (Calera cuadrícula 2, Holoceno tardío), 4 ejs.; FCS-IN CAL 278 (Calera cuadrícula 2, Holoceno tardío), 27 ejs.; FCS-IN 
CAL 303 (Calera cuadrícula 2, Holoceno tardío), 13 ejs. Materiales comparados. MACN-In 36.354. Formosa: Río Pilagá; MLP 10934. Berisso, Buenos Aires; MLP 11782. Balneario, Quilmes, Buenos Aires.

Dimensiones del material fotografiado. Altura: $7 \mathrm{~mm}$. Distribución actual en Argentina. Provincias de Formosa, Corrientes, Chaco, Santa Fe, Entre Ríos y Buenos Aires.

Distribución estratigráfica. Holoceno (primer registro ilustrado de esta especie).

Registros en el Cuaternario de la Argentina. Sitio Calera (Steffan, 2009).

Familia PLANORBIDAE Rafinisque, 1815

Drepanotrema Fischer \& Crosse, 1880

Drepanotrema kermatoides (d'Orbigny, 1835)

(Figuras 3Ea; Tablas 3,4)

1835 Planorbis kermatoides d'Orbigny, p. 27.

1991 Drepanotrema kermatoides Rumi, p. 30, fig. 1c; fig. 6b; fig. 50,b.

Materiales recuperados. FCS-IN CAL 236 (Calera cuadrícula 1, Holoceno tardío), 6 ejs.; FCS-IN CAL 242 (Calera cuadrícula 2, Holoceno tardío), 5 ejs.; FCS-IN CAL 264 (Calera cuadrícula 2, Holoceno tardío), 161 ejs.; FCS-IN CAL 286 (Calera cuadrícula 2, Holoceno tardío); 1.588 ejs.

Material comparado. MACN-In 29358. Paranacito, islas del Delta.

Dimensiones del material fotografiado. Diámetro mayor: $3,5 \mathrm{~mm}$.

Distribución actual en Argentina. sector occidental de las provincias de Misiones, Corrientes, Chaco, Santa Fe, Entre Ríos y Buenos Aires.

Distribución estratigráfica. Holoceno (primer registro cuaternario de la especie).

Biomphalaria Preston, 1910

Biomphalaria peregrina (d'Orbigny, 1835)

(Figuras 3Fc,d; Tablas 3, 4)

1835 Planorbis peregrinus d'Orbigny, p. 26, pl. 44, figs. 13-16. 2006 Biomphalaria peregrina Gutiérrez Grégoric et al., p. 53, fig. 14 .

Materiales recuperados. FCS-IN RQG 10 (Paso Otero 1, Holoceno medio), 106 ejs.; FCS-IN RQG 6 (Paso Otero 1, Holoceno tardío), 48 ejs.; FCS-IN RQG 62 (Paso Otero 3, Holoceno medio), 1 ej.; FCS-IN RQG 116 (Paso Otero 3, Holoceno medio), 98 ejs.; FCS-IN RQG 162 (Paso Otero 3, Holoceno tardío), 52 ejs.; FCS-IN C LA 215 (Campo Laborde, Holoceno temprano), 9 ejs.; FCS-IN CAL 245 (Calera cuadrícula 2, Holoceno tardío), 15 ejs.; FCS-IN CAL 253 (Calera cuadrícula 2, Holoceno tardío), 512 ejs.; FCSIN COR 336 (Cortaderas, Holoceno tardío), 67 ejs.; FCS-IN
ACOR 361 (Arroyo Cortaderas, Holoceno tardío), 462 ejs. Materiales comparados. 1 Lectotipo (NHM 1854.12.4.2671a). MLP 7018-1; 7018-2. Río Abra Pampa o Miraflores, Abra Pampa, Jujuy. MLP 7177. Laguna La Copeta, Guaminí, Buenos Aires; MLP 7357. Arroyo Belisario, Sierra de la Ventana, Buenos Aires; MLP 7793. Río Savallen, Salta.

Dimensiones del material fotografiado. Diámetro mayor: $4,8 \mathrm{~mm}$. Distribución actual en Argentina. En casi toda la Argentina, hasta la provincia de Chubut.

Distribución estratigráfica. Holoceno.

Registros en el Cuaternario de Argentina. Holoceno, Mendoza, arroyo La Estancada (Tunuyán) (De Francesco et al., 2007); Miembro Río Salado de la Formación Luján (este trabajo).

Antillorbis Harry \& Hubendick, 1964

Antillorbis nordestensis (Lucena, 1954)

(Figura 3Ga; Tablas 3,4)

1954 Tropicorbis nordestensis Lucena, p. 329, fig. 1.

2006 Antillorbis nordestensis Gutiérrez Grégoric et al., p. 53, fig. 12 .

Materiales recuperados. FCS-IN CAL 233 (Calera cuadrícula 2, Holoceno tardío), 1 ej.; FCS-IN CAL 234 (Calera cuadrícula 2, Holoceno tardío), 2 ejs.; FCS-IN CAL 254 (Calera cuadrícula 2, Holoceno tardío), 15 ejs.

Materiales comparados. MACN-In 17.854-1. Buenos Aires, Rosas; MLP 7278. Misiones: Aristóbulo del Valle, arroyo Cuña Pirú; MLP 7015- 5. Isla Martín García, Buenos Aires. Dimensiones del material fotografiado. Diámetro mayor: 2,7 mm.

Distribución actual en Argentina. Provincias de Santa Fe y Buenos Aires.

Distribución estratigráfica. Holoceno-Actualidad.

Registros en el Cuaternario de Argentina. Holoceno (primer registro fósil del Cuaternario para Argentina).

Familia ANCYLIDAE Rafinisque, 1815

Uncancylus Pilsbry, 1913

Uncancylus concentricus (d'Orbigny, 1835)

(Figuras 3Hc,e; Tablas 3,4)

1835 Ancylus concentricus d'Orbigny, p. 24

1837 Ancylus concentricus d'Orbigny, p. 354, pl. 42, figs. 18-21. 2006 Uncancylus concentricus Gutiérrez Grégoric et al., p. 53 , fig. 9 .

Materiales recuperados. FCS-IN CAL 257 (Calera cuadrícula 2, Holoceno tardío), 2 ejs.; FCS-IN CAL 295 (Calera cuadrícula 2, Holoceno tardío), 1 ej.; FCS-IN ACOR 341 (Arroyo Cortaderas, Holoceno tardío), 1 ej.

Materiales comparados. 7 Sintipos (NHM 1854.12.4.2791b). MLP 4548. Río Quequén, Buenos Aires; MLP 7340. Charca de la ruta 16, llegando a San Pedro, Misiones. 
Dimensiones del material fotografiado. Altura: $5 \mathrm{~mm}$. Distribución actual en Argentina. En casi todo el país, incluyendo los lagos de la región patagónica.

Distribución estratigráfica. Holoceno.

Registros en el Cuaternario de Argentina. Holoceno. Buenos Aires: Pilar (Fucks et al., 2005). En paleosuelo en sedimentos del Miembro Río Salado de la Formación Luján (Holoceno) del área Interserrana de las Sierras Bayas, sitio arqueológico Arroyo Seco.

Suborden STYLOMMATOPHORA Schmidt, 1855 Familia SCOLODONTIDAE Baker, 1925

Miradiscops Baker, 1925

Miradiscops brasiliensis (Thiele, 1927)

(Figura 4Da; Tablas 3,4)

1927 Microhappia brasiliensis Thiele, p. 320, fig. 17a-c. 1959 Miradiscops (Punctodiscops) brasiliensis Zilch, p. 545. 2005 Miradiscops brasiliensis Miquel \& Steffan, p. 495.

Materiales recuperados. FCS-IN C LA 224 (Campo Laborde, Holoceno temprano), 1 ej.; FCS-IN RQG 80 (Paso Otero 5, Holoceno temprano), 10 ejs.; FCS-IN RQG 7 (Paso Otero 1, Holoceno medio), 190 ejs.; FCS-IN RQG 125 (Paso Otero 5, Holoceno medio), 9 ejs.; FCS-IN RQG 3 (Paso Otero 1, Holoceno tardío), 168 ejs.; FCS-IN RQG 53 (Paso Otero 5, Holoceno tardío), 3 ejs.; FCS-IN RQG 113 (Paso Otero 3, Holoceno tardío), 10 ejs.; FCS-IN QS 194 (Quequén Salado, Holoceno tardío), 2 ejs.; FCS-IN CAL 249 (Calera cuadrícula 2, Holoceno tardío), 63 ejs.; FCS-IN COR 333 (Cortaderas, Holoceno tardío), 43 ejs.; MACN-In 36.061 (Paso Otero 1, río Quequén Grande), 2 ejs.; MACN-Pi 4.734 (Buenos Aires: Paso Otero 1, río Quequén Grande), 2 ejs.

Material comparado. MACN-In 30424. Río de las Conchas, Merlo, Buenos Aires.

Dimensiones del material fotografiado. Diámetro mayor: $1,8 \mathrm{~mm}$.

Distribución actual en Argentina. Presente en la Mesopotamia y en el norte y centro del país.

Distribución estratigráfica. Holoceno.

Registros en el Cuaternario de Argentina. Holoceno, Buenos Aires, Paso Otero, sitio Calera, arroyo Cortaderas (este trabajo); Entre Ríos (Aguirre et al., 2010).

Familia CHAROPIDAE Hutton, 1884

Zilchogyra Weyrauch, 1965

Zilchogyra costellata (d'Orbigny, 1835)

(Figura 4Aa; Tablas 3,4)

1835 Helix costellata d'Orbigny, p. 252.

2004 Zilchogyra costellata (d’Orbigny); Miquel et al., p. 925.

Materiales recuperados. FCS-IN RQG 104 (Paso Otero 3, Holoceno tardío), 1 ej.; FCS-IN RQG 140 (Paso Otero 3,
Holoceno tardío), 10 ejs.; FCS-IN RQG 12 (Paso Otero 1, Holoceno tardío), 26 ejs.; FCS-IN RQG 17 (Paso Otero 1, Holoceno tardío), 2 ej.

trabajo).

Materiales comparados. 1 Sintipo (NHM 1854.12.4.69). MACN-In 13.103. Buenos Aires: Miramar, arroyo Durazno; MACN-In 16.529. Buenos Aires, Tandil.

Dimensiones de los materiales fotografiados. Diámetro mayor: $2,3 \mathrm{~mm}$ y $2,6 \mathrm{~mm}$.

Distribución actual en Argentina. Provincia de Buenos Aires.

Distribución estratigráfica. Holoceno.

Registros en el Cuaternario de Argentina. Holoceno, Lobería, río Quequén Grande (este trabajo).

Retidiscus Fonseca \& Thomé, 1995

Retidiscus reticulatus Fonseca \& Thomé, 1995

(Figura 4Ba; Tablas 3, 4)

1995 Retidiscus reticulatus Fonseca \& Thomé, p. 55, figs. 1-6. 2005 Retidiscus reticulatus Miquel \& Steffan, p. 495-500, Fig. 2.

Materiales recuperados. FCS-IN RQG 2 (Paso Otero 1, Holoceno tardío), 59 ejS.; FCS-IN RQG 105 (Paso Otero 3, Holoceno tardío), 2 ejS.; FCS-IN RQG 124 (Paso Otero 3, Holoceno tardío), 17 ejS.; FCS-IN RQG 158 (Paso Otero 3, Holoceno tardío), 329 ejs.; FCS-IN CAL 272 (Calera cuadrícula 2, Holoceno tardío), 56 ejs.; FCS-IN COR 327 (Cortaderas, Holoceno tardío), 5 ejs.; FCS-IN ACOR 356 (Arroyo Cortaderas, Holoceno tardío); 2 ejs.

Material comparado. MACN-In 34.197. Brasil, Rio Grande do Sul, Viamão, Parque Saint-Hilaire.

Dimensiones del material fotografiado. Diámetro mayor: $1,6 \mathrm{~mm}$.

Distribución actual en Argentina. Provincia de Buenos Aires. Distribución estratigráfica. Holoceno.

Familia PUNCTIDAE Morse, 1864

Paralaoma Iredale, 1913

Paralaoma servilis (Shuttleworth, 1852)

(Figura 4Ca; Tablas 3,4)

1852 Helix servilis Shuttleworth, p. 40.

2003 Paralaoma servilis Neubert \& Gosteli, p. 49, pl. 15, fig. 3. 2007 Paralaoma servilis Miquel et al., p. 207, fig. 3.

Material recuperado. FSC-IN-RQG 173 (Río Quequén Grande, Buenos Aires); 1 ej. Recuperado de la recolección superficial de la planicie de inundación del río.

Materiales comparados. 6 Sintipos ZMZ 503343. MACN-In 36.107. Formosa, Reserva ecológica El Bagual.

Dimensiones del material fotografiado. Diámetro mayor: $1,8 \mathrm{~mm}$. 
Distribución estratigráfica. Actual. No existen registros fósiles de esta especie para la Argentina, por ser una especie introducida (Miquel \& Steffan, 2005; Miquel et al., 2007).

Familia SUCCINEIDAE Beck, 1837

Succinea Draparnaud, 1801

Succinea meridionalis d'Orbigny, 1837

(Figuras 4Ec,d; Tablas 3,4)

1837 Succinea meridionalis d'Orbigny, p. 251.

1973 Succinea meridionalis Fernandez \& Castellanos, p. 269, lám. I, fig. 3.

Materiales recuperados. FCS-IN RQG 78 (Paso Otero 5, Holoceno temprano), 210 ejs.; FCS-IN RQG 223 (Campo Laborde, Holoceno temprano), 308 ejs.; FCS-IN RQG 8 (Paso Otero 1, Holoceno medio), 11 ejs.; FCS-IN RQG 4 (Paso Otero 1, Holoceno tardío), 63 ejs.; FCS-IN RQG 19 (Paso Otero 1, Holoceno tardío), 1 ej.; FCS-IN RQG 107 (Paso Otero 3, Holoceno tardío), 31 ejs.; FCS-IN QS 197 (Quequén Salado, Holoceno tardío), 939 ejs.; FCS-IN CAL 275 (Calera cuadrícula 2, Holoceno tardío), 12 ejs.; FCS-IN COR 334 (Cortaderas, Holoceno tardío), 108 ejs.; FCS-IN ACOR 358 (Arroyo Cortaderas, Holoceno tardío), 35 ejs.

Materiales comparados. MLP 2157. Córdoba: Bajo Chico; MLP 1122. Buenos Aires: Médanos de Miramar, Miramar; MLP 10611. Buenos Aires. Quequén.

Dimensiones del material fotografiado. Altura: $3,5 \mathrm{~mm}$. Distribución actual en Argentina. Norte y centro del país, hasta el norte de Patagonia.

Distribución estratigráfica. Pleistoceno-Holoceno.

Registros en el Cuaternario de la Argentina. Holoceno del sudoeste de Entre Ríos (Aguirre et al., 2010); Holoceno: Mendoza (De Francesco \& Diequez, 2006); Buenos Aires: arroyo Tapalqué (Figini et al., 1998), Paso Otero, Salto de Piedra, río Quequén Grande (Bonadonna et al., 1999), sitios Cortaderas y Campo Laborde (este trabajo).

Omalonyx d'Orbigny, 1835

Omalonyx unguis d'Orbigny, 1835

(Figuras 4Fc,d; Tablas 3,4)

1835 Helix (Cochlohydra) unguis d'Orbigny, p. 2.

1841 Succinea (Omalonyx) unguis d'Orbigny, p. 229, pl. 22, figs. 1-7.

1995 Omalonix unguis Castellanos \& Landoni, p. 778.

Materiales recuperados. FCS-IN CAL 250 (Calera cuadrícula 2, Holoceno tardío), 2 ejs.; FCS-IN CAL 261 (Calera cuadrícula 2, Holoceno tardío), 7 ejs.; FCS-IN CAL 274 (Calera cuadrícula 2, Holoceno tardío), 3 ejs.

Materiales comparados. MACN-In 14.860. Buenos Aires, Rosas. MACN-In 27.241. Buenos Aires, laguna de Navarro; MLP 9599. Corrientes, Manantiales.
Dimensiones del material fotografiado. Altura: $8 \mathrm{~mm}$. Distribución actual en Argentina. Provincias de Formosa, Corrientes, Chaco, Santa Fe, Entre Ríos y Buenos Aires.

Distribución estratigráfica. Holoceno.

Registros en el Cuaternario de Argentina. Holoceno: sitio Calera (primer registro fósil de esta especie para el Holoceno y para la Argentina).

Clase BIVALVIA Linné, 1758

Orden VENEROIDA Adams \& Adams, 1856

Familia SPHAERIIDAE Deshayes, 1854

Pisidium Pfeiffer, 1821

Pisidium sp.

(Figuras 3Ic,d; Tablas 3,4)

Materiales recuperados. FCS-IN RQG 243 (Paso Otero 5, Holoceno temprano), 2 ejs.; FCS-IN CAL 258 (Calera cuadrícula 2, Holoceno tardío), 396 ejs.; FCS-IN CAL 304 (Calera cuadrícula 2, Holoceno tardío), 75 ejs.; FCS-IN CAL 312 (Calera cuadrícula 2, Holoceno tardío), 28 ejs.

Material comparado. Pisidium vile Pilsbry, 1897: MLP 5063. Río Santiago, Ensenada, Buenos Aires.

Dimensiones del material fotografiado. Largo: $2,5 \mathrm{~mm}$. Distribución actual en Argentina. Género de distribución mundial.

Distribución estratigráfica. Holoceno.

Registros en el Cuaternario de la Argentina. Holoceno, Buenos Aires, Calera (Steffan, 2009). Primer registro fósil para el Holoceno del área Interserrana.

Abundancia relativa de los taxones y descripciones tafonômicas. Cualitativamente, el contenido malacológico de los tres sectores analizados es similar (Tablas 5a,b). Sin embargo, el sitio Calera aporta la mayor abundancia de ejemplares de todos los taxones recuperados, con predominio de Drepanotrema kermatoides. Por el contrario, la menor abundancia se registra en los sitios Campo Laborde y Paso Otero 5 (Tabla 5b).

\section{Sector Río Quequén Grande}

Paso Otero 1 ( 9,6-2,9 ka AP). Heleobia parchappii es la especie más abundante (Figura 5). De los parámetros tafonómicos evaluados, las conchillas de H. parchappii y Biomphalaria peregrina son las más afectadas por la fragmentación. $\mathrm{Al}$ considerar la fragmentación en sentido vertical (cronológico) en el perfil se destaca un elevado porcentaje (84\%) en el paleosuelo Puesto Berrondo (Unidad II superior) (Figura 1a; Tabla 2), donde también se observa la máxima abundancia de $H$. parchappii. Estas características han sido interpretadas como indicativas de un flujo de mayor energía, relacionado probablemente por con eventos de inundación ( 4,9-3 ka AP) (ver Figura 1; Tabla 2) (Steffan, 2007). Sin embargo, la mayor depositación química $\left(\mathrm{CaCO}_{3}\right)$ se registra para el mismo sector, por lo cual se infiere la posterior desecación del material depositado. La precipitación de carbonato de calcio, como se comentó previamente se vincula con humedad insuficiente. 


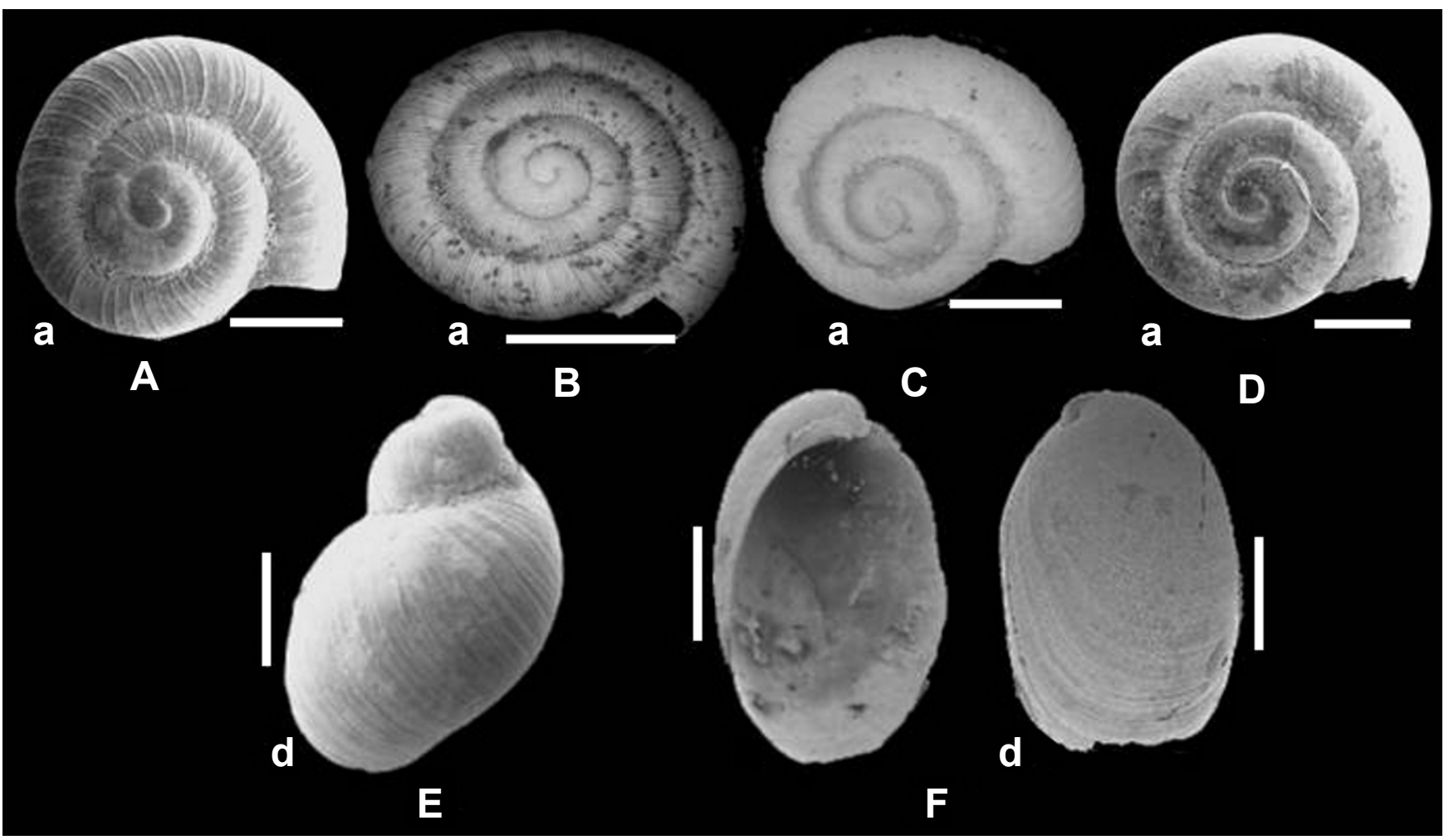

Figura 4. Gastropodos terrestres: A, Zilchogyra costellata, Paso Otero 1, Holoceno; B, Retidiscus reticulatus, Paso Otero 1, Holoceno (modificada de Miquel \& Steffan, 2005); C, Paralaoma servilis, La Dulce; D, Miradiscops brasiliensis, Paso Otero 1, Holoceno; E, Succinea meridionalis, Paso Otero 1, Holoceno; F, Omalonyx unguis, Calera, Holoceno tardío. Abreviaturas: a, vista dorsal; b, vista umbilical; c, vista apertural d, vista adapertural. Escalas: $A, C, E=1 \mathrm{~mm} ; B, D=500 \mu \mathrm{m} ; F=200 \mu \mathrm{m}$.

Figure 4. Terrestrial gastropods: A, Zilchogyra costellata, Paso Otero 1, Holocene; B, Retidiscus reticulatus, Paso Otero 1, Holocene (modified of Miquel \& Steffan, 2005); C, Paralaoma servilis, La Dulce; D, Miradiscops brasiliensis, Paso Otero 1, Holocene; E, Succinea meridionalis, Paso Otero 1, Holocene; F, Omalonyx unguis, Calera, late Holocene. Abbreviations: a, dorsal view; b, umbilical view; c, apertural view d, adapertural view. Scale bars: $A, C, E=1 \mathrm{~mm} ; B, D=500 \mu \mathrm{m} ; F=200 \mu \mathrm{m}$.

Paso Otero 3 ( 4,7-1,5 ka AP). El taxón más abundante es Heleobia parchappii (Figura 6A; Tabla 3). El análisis tafonómico permite observar una fragmentación del $29 \%$ de las conchillas, corrasión en un $24 \%$, el $78 \%$ de las conchillas presenta depositación química $\left(\mathrm{CaCO}_{3}, \mathrm{Mn}\right)$ y $54 \%$ de incrustación interna. En sentido vertical a lo largo del perfil, entre las variables tafonómicas se destacan 1) el alto grado de fragmentación y corrasión entre $\sim 2,2$ ka AP y 2,1 ka AP; 2) el $\mathrm{CaCO}_{3}$ es más frecuente en los niveles inferiores, sobre todo entre 3 y 2,2 $\mathrm{ka}$ AP. La mayor abundancia de especies acuáticas se registra en $\sim 2,1-2$ ka AP (Figura 6A).

Paso Otero 5 ( 10,4-2,5 ka AP). La mayor abundancia corresponde a Succinea meridionalis y se registra en la base del perfil (Holoceno temprano) (Figura 6a; Tabla 3). En general se observa un buen estado de preservación de las conchillas: la fragmentación observada $(17 \%)$ es la más baja de los 3 sitios de la localidad arqueológica Paso Otero y corresponde a Heleobia parchappii hacia el tope de la secuencia estratigráfica (ca. 2,5 ka AP.); la corrasión es baja (20\%). En particular para $H$. parchappii y $S$. meridionalis en la base del perfil se observa un predominio de conchillas con signos de depositación química $\left(\mathrm{CaCO}_{3}\right)(83 \%)$ y la incrustación interna es de $91 \%$.

\section{Sector sudoeste del área Interserrana}

Cortaderas (Holoceno tardío). El taxón más abundante es Heleobia parchappii (Figura 6B; Tabla 3). Se destaca la fragmentación $(22 \%)$ en algunos ejemplares de $H$. parchappii, especialmente con posterioridad a los ca. 2,2 ka AP (Figura 6B). Esto coincide con el nivel de mayor abundancia del perfil estratigráfico y con los mayores porcentajes de incrustación y depositación química. Los valores observados son $15 \%$ de corrasión, $39 \%$ de depositación química y $22 \%$ de incrustación interna.

Curso inferior del río Quequén Salado (Holoceno temprano-tardío). La mayor abundancia corresponde a Succinea meridionalis, especialmente en la Unidad 3b (U3b) (Figura 6B; Tabla 3). Se observa una muy baja fragmentación $(15 \%)$ y corrasión $(14 \%)$, la depositación química $\left(\mathrm{CaCO}_{3}\right)$ es del $49 \%$, con mayor importancia en $S$. meridionalis de la U 3 b y la incrustación interna es del $32 \%$.

\section{Sector cuenca superior del arroyo Tapalqué}

Campo Laborde (Holoceno temprano). La especie más abundante es Succinea meridionalis concentrada en la base del perfil (ca. 8 ka AP) (Figura 6C). En cuanto al estado de 


\section{Cuenca media del río Quequén Grande}

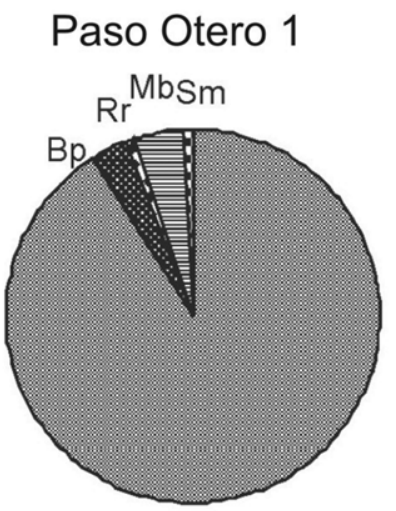

$\mathrm{Hp}$
Paso Otero 5

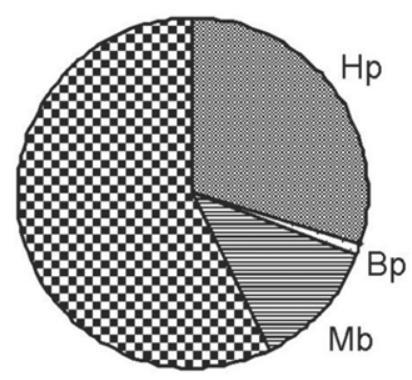

$\mathrm{Sm}$
Paso Otero 3

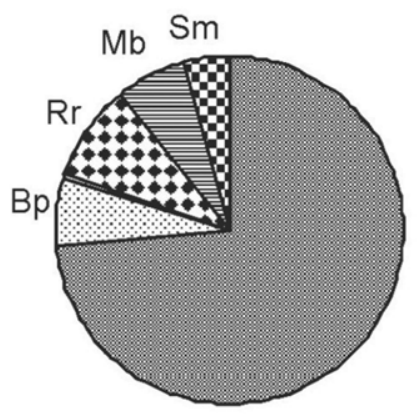

$\underline{\mathrm{Hp}}$

\section{Sudoeste del área Interserrana}

\section{Cortaderas}

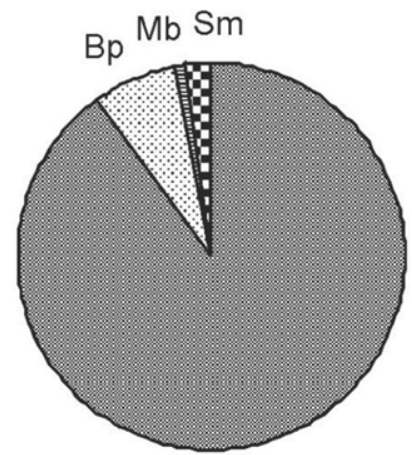

$\mathrm{Hp}$

\section{Quequén Salado}

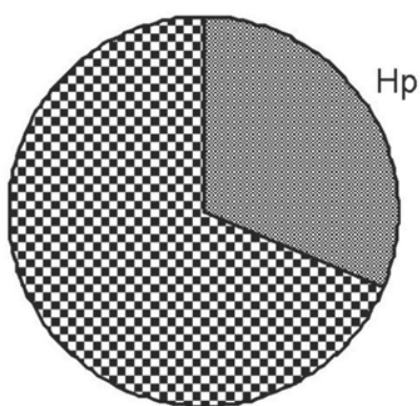

$\mathrm{Sm}$

\section{Cuenca superior del arroyo Tapalqué}

\section{Campo Laborde}

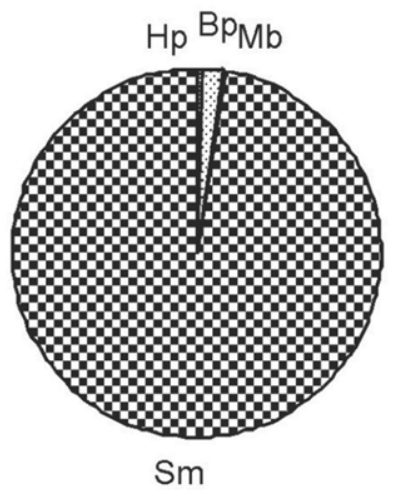

Calera

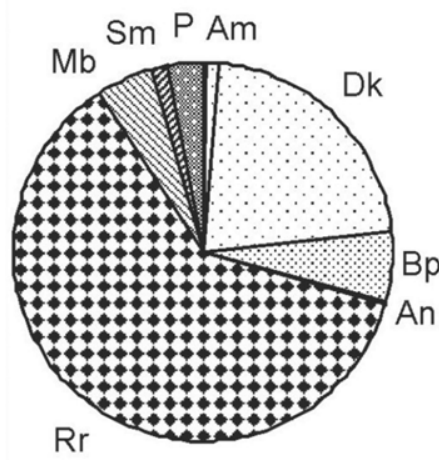

Figura 5. Análisis cuantitativo de la malacofauna recuperada en las áreas relevadas. I, cuenca media del río Quequén Grande; II, sudoeste del área Interserrana; III, cuenca superior del arroyo Tapalqué. Abreviaturas: Rr, Retidiscus reticulatus; Mb, Miradiscops brasiliensis; Sm, Succinea meridionalis; Hp, Heleobia parchappii; Bp, Biomphalaria peregrina; An, Antillorbis nordestensis; Dk, Drepanotrema kermatoides; Am, Aplexa minor, P, Pisidium sp.

Figure 5. Quantitive analysis of malacofauna recovered in the areas surveyed. I, middle basin of the Quequén Grande river cuenca media del río; II, southwest Interserrana area; III, upper basin of the Tapalqué stream. 
preservación se observa solo un $15 \%$ de fragmentación en S. meridionalis en los niveles inferiores del perfil (Holoceno temprano), la corrasión es muy baja (15\%), solo el $30 \%$ presenta depositación química, mientras que la incrustación interna es del 59\%. Si bien la incrustación y la depositación química se vinculan con alteraciones, es necesario ampliar que los valores mínimos de incrustación se registran entre 115-140 m (Holoceno temprano) y los máximos valores entre 90-115 m (tope de la secuencia). De manera similar, los valores mínimos de depositación química se registran entre 110-140 m y los valores máximos se registran en 105-110 m y 140-145 m. Por lo tanto, dicha muestra es significativa para el análisis del Holoceno temprano.

Calera (ca. 3,3-2 ka AP) (Tabla 2). La mayor abundancia de ejemplares se observa en Drepanotrema kermatoides y Retidiscus reticulatus (Tabla 3). Se registra una elevada fragmentación (49\%), particularmente en los taxones acuáticos de concha muy delgada como "Aplexa" minor, Drepanotrema kermatoides, Uncancylus concentricus y Pisidium sp. La corrasión es la más elevada de todos los sitios (26\%), especialmente en Succinea meridionalis. La presencia de depositación química es del $37 \%$ y la incrustación interna es mínima (9\%). Estos bajos valores se observan con frecuencia en ambientes acuáticos. Conjuntamente, estos atributos tafonómicos sugieren que parte de los moluscos fueron afectados in situ por el agua mientras otros como $S$. meridionalis pudieron ingresar a través del agua que escurre en manto.

\section{Indices y análisis estadístico}

Por otro lado, de acuerdo con los resultados obtenidos mediante la aplicación del índice de riqueza específica (d) de Margalef a los sitios analizados, el valor índice más elevado se registra en Calera, donde se identificaron un total de 11 taxones, mientras que el de menor riqueza es Quequén Salado, con sólo tres taxones (Tabla 5b). El índice de diversidad (H") de Shannon-Wiener muestra los máximos valores para Calera y el menor valor en Campo Laborde. En orden decreciente de diversidad se ubican Paso Otero 5, Paso Otero 3, Quequén Salado, mientras que en los sitios Paso Otero 1 y Cortaderas se documentaron valores similares de diversidad.

La asociación de sitios sobre la base del contenido malacológico, obtenido a partir del cluster analysis (CA) aplicando el coeficiente de asociación de Jaccard muestra una clara discriminación del sitio Calera del resto de los sitios (con un valor de similitud de 0,38) (Figura 7). Para el resto de los sitios es posible reconocer dos grupos (I y II) ligados por un valor de similitud de 0,50. En el primer grupo (I) el sitio Cortaderas está ligado por un valor de similitud de 0,54 con los sitios Paso Otero 3 y Paso Otero 1, que están vinculados por una similitud de 0,88 . En el otro grupo (II) se registran los sitios Paso Otero 5 y Campo Laborde con el valor de máxima similitud (1) y ambos con Quequén Salado comparte una similitud de 0,75 .

El análisis de coordenadas principales (PCA, Principal Coordinate Analysis) arrojó resultados coincidentes y permite observar la separación espacial Calera del resto de los sitios arqueológicos estudiados. Asimismo, se observan los sitios Paso Otero 1, 3 y Cortaderas agrupados, mientras que a mayor distancia se observa Quequén Salado (Figura 7). El Coeficiente de Correlación Cofenética es de 0,94 , por lo tanto la distorsión que se produce sobre los datos de la matriz de similitud es mínima. Los agrupamientos de sitios, tanto por CA como por PCA, corroboran los dos grupos principales y la separación de Cortaderas.

\section{DISCUSIÓN}

En la Provincia de Buenos Aires, la malacofauna dulciacuícola moderna se caracteriza por ser pobre, disminuyendo su diversidad específica hacia el sur y el oeste (Landoni, 1992); sin embargo, ese panorama no resulta ser similar para la malacofauna terrestre, ya que las áreas de Tandilia y Ventania, en el sudeste bonaerense, exhiben la mayor riqueza de especies de gastrópodos de toda la provincia geográfica (Fernández, 1973). Por el contrario, en este trabajo, si bien se debe reconocer que se analizan sectores de valles, se observan diferencias con respecto a la diversidad de la malacofauna moderna debido a que el registro fósil de taxones de moluscos dulciacuícolas es más diverso (ocho taxones) que la malacofauna terrestre (Miradiscops brasiliensis, Zilchogyra costellata, Retidiscus reticulatus). La lista taxonómica se complementa con dos taxones higrófilos (Succinea meridionalis, Omalonyx unguis) y uno introducido (Paralaoma servilis).

En relación con la distribución estratigráfica de los taxones identificados, los rangos temporales abarcan desde el Holoceno temprano hasta la actualidad (Tabla 4). Los taxones Heleobia parchappii, Chilina parchappii y Lymnaea viator cuentan con registros documentados desde el Pleistoceno. En el Holoceno se identificaron por primera vez fósiles de "Aplexa" minor, Drepanotrema kermatoides, Antillorbis nordestensis (Lucena, 1954), Uncancylus concentricus, Zilchogyra costellata, Omalonyx unguis y Pisidium sp.

Teniendo en cuenta los resultados de agrupamientos estadísticos obtenidos para los sitios, tanto por CA como por PCA, una explicación posible seria la diversidad taxonómica desde una perspectiva temporal. La composición de la malacofauna característica de la etapa inicial del Holoceno temprano se encuentra representada mayoritariamente Succinea meridionalis, especie de hábitos higrófilos o sectores pantanosos (Figuras 5A-C; Tabla 3). Posteriormente al Holoceno temprano se observa una mayor abundancia de los taxones fluviales y lacustres (Heleobia parchappii, Biomphalaria peregrina) para la mayoría de los sitios analizados. Sin embargo, la malacofauna del sitio Calera presenta diferencias respecto de los anteriores, presentando mayor abundancia de Retidiscus reticulatus durante un lapso acotado a $\sim 3,3$ ka AP y 1,7 ka AP (Figura 6C).

Variaciones de la malacofauna durante el Holoceno temprano (ca. 10 - 6,5 ka AP): Paso Otero 5 y Campo Laborde

El registro malacológico de los sitios Paso Otero 5 ( $c a$. 10,4 ka AP) y Campo Laborde es poco abundante en el techo del Miembro Guerrero (límite Pleistoceno tardíoHoloceno temprano) y en la base de los depósitos arenolimosos del Miembro Río Salado (Holoceno temprano) 
I. Cuenca media del río Quequén Grande

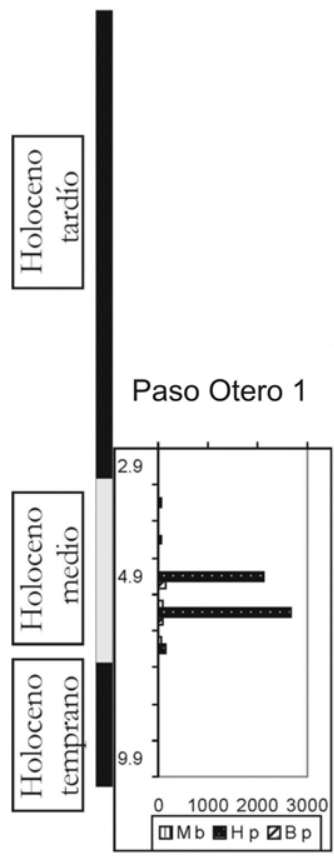

A
II. Sudoeste del área Interserrana

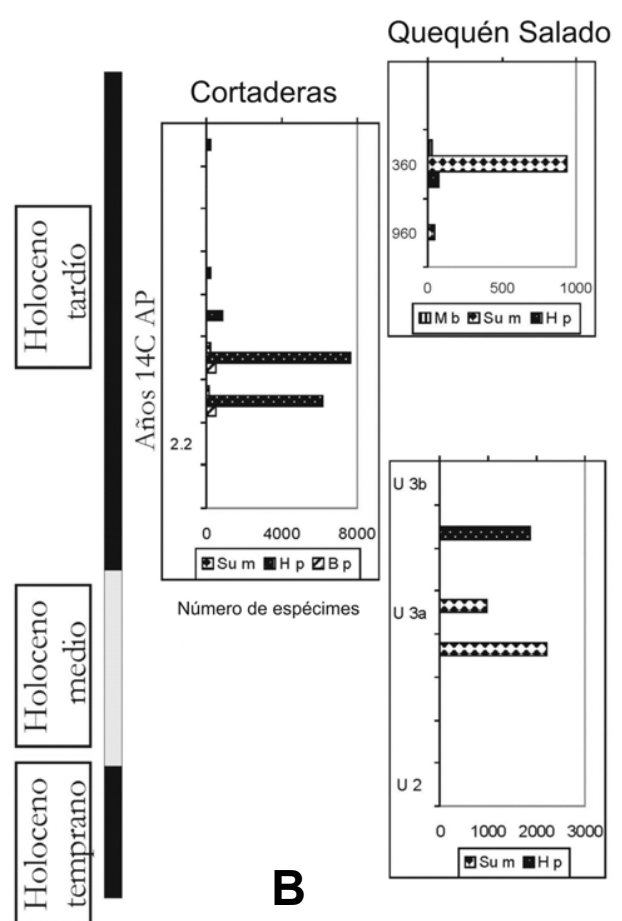

\section{Cuenca superior del arroyo Tapalqué}

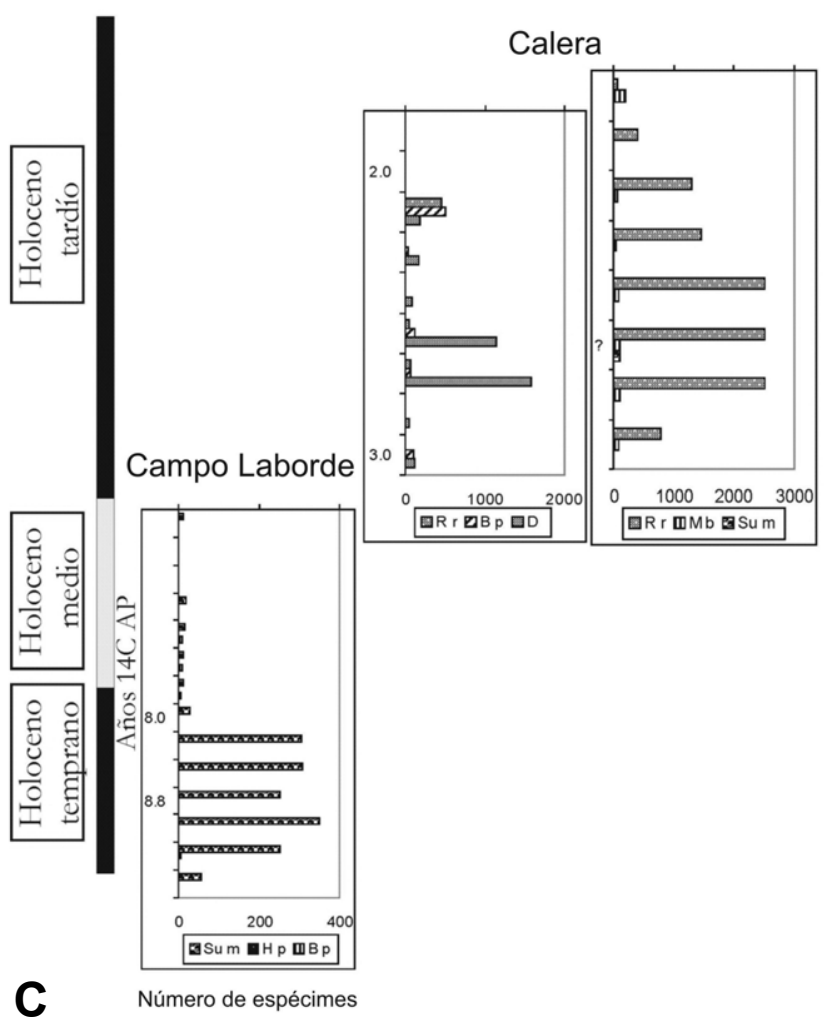

Figura 6. Abundancia relativa de moluscos durante el Holoceno en: A, cuenca media del río Quequén Grande; $\mathbf{B}$, sudoeste del área Interserrana; C, cuenca superior del arroyo Tapalqué. Se ilustran los tres taxones más abundantes de cada perfil analizado.

Figure 6. Relative abundance of mollusks during the Holocene: A, Middle basin of Quequén Grande river; B, southwest Interserrana area; C, upper basin of the Tapalqué stream. The three most abundant taxa are shown for each profile analyzed. 
Tabla 5. Síntesis del análisis cuantitativo de la malacofauna recuperada: 5a, en los tres sectores estudiados; 5 b, en los siete sitios arqueológicos analizados. Abundancia relativa de especies, índices de riqueza específica (d) de Margalef y de diversidad (H") de ShannonWiener de los sitos estudiados.

Table 5. Summary of quantitative analysis of malacofauna recovered: $\mathbf{5 a}$, in the three studied sectors; $\mathbf{5 b}$, in the seven archaeological sites analyzed. Relative abundance of species, specific richness (d) of Margalef and diversity (H”) of Shannon-Wiener index of studied sites.

\begin{tabular}{|c|c|c|c|c|c|c|}
\hline Sector & Sitio & $\begin{array}{c}\text { Columna de } \\
\text { muestreo en sitios }\end{array}$ & $\begin{array}{l}\text { Perfiles de muestreo } \\
\text { en barrancas }\end{array}$ & $\begin{array}{c}\text { Material } \\
\text { de Cernidor }\end{array}$ & $\begin{array}{c}\text { Total de ejemplares } \\
\text { por sitio }\end{array}$ & $\begin{array}{l}\text { Total de ejemplares } \\
\text { por sector }\end{array}$ \\
\hline \multirow{3}{*}{$\begin{array}{l}\text { I- Cuenca media del } \\
\text { río Quequén Grande }\end{array}$} & PO 1 & 13.577 & & & 13.577 & \multirow{3}{*}{28.827} \\
\hline & PO 3 & 14.173 & & & 14.173 & \\
\hline & PO 5 & 1.077 & & & 1.077 & \\
\hline \multirow{2}{*}{ II- Sudoeste del area Interserrana } & COR & 2.801 & 13.917 & & 16.718 & \multirow{2}{*}{22.891} \\
\hline & QS & 5.071 & 1.102 & & 6.173 & \\
\hline \multirow{3}{*}{$\begin{array}{l}\text { III- Cuenca superior } \\
\text { del arroyo Tapalqué }\end{array}$} & $\mathrm{CL}$ & 40 & & 1.632 & 1.672 & \multirow{2}{*}{21.370} \\
\hline & $\mathrm{CA}$ & & 12.407 & 7.291 & 19.698 & \\
\hline & \multicolumn{4}{|c|}{ Total de ejemplares } & \multicolumn{2}{|c|}{73.088} \\
\hline
\end{tabular}

\begin{tabular}{|c|c|c|c|c|c|}
\hline Localidad & Taxones & $\mathrm{n}^{\mathrm{o}}$ de individuos & $\mathrm{n}^{\mathrm{o}}$ de especies & $\mathrm{d}$ & $\mathrm{H}^{\prime \prime}$ \\
\hline \multirow{8}{*}{ PASO OTERO 1} & H. parchappii & 12.342 & & & \\
\hline & Ch. parchappii & 11 & & & \\
\hline & B. peregrina & 419 & & & \\
\hline & Z. costellata & 31 & & & \\
\hline & R. reticulatus & 95 & & & \\
\hline & M. brasiliensis & 564 & & & \\
\hline & S. meridionalis & 115 & & & \\
\hline & Total & 13.577 & 7 & 0,63 & 0,18 \\
\hline \multirow{8}{*}{ PASO OTERO 3} & H. parchappii & 10.445 & & & \\
\hline & Ch. parchappii & 1 & & & \\
\hline & B. peregrina & 864 & & & \\
\hline & Z. costellata & 60 & & & \\
\hline & R. reticulatus & 1.275 & & & \\
\hline & M. brasiliensis & 929 & & & \\
\hline & S. meridionalis & 599 & & & \\
\hline & Total & 14.173 & 7 & 0,63 & 0,41 \\
\hline \multirow{5}{*}{ PASO OTERO 5} & H. parchappii & 321 & & & \\
\hline & B. peregrina & 11 & & & \\
\hline & M. brasiliensis & 129 & & & \\
\hline & S. meridionalis & 616 & & & \\
\hline & Total & 1.077 & 4 & 0,43 & 0,43 \\
\hline \multirow{8}{*}{ CORTADERAS } & H. parchappii & 15.041 & & & \\
\hline & L. viator & 9 & & & \\
\hline & B. peregrina & 1.166 & & & \\
\hline & $U$. concentricus & 1 & & & \\
\hline & R. reticulatus & 13 & & & \\
\hline & M. brasiliensis & 106 & & & \\
\hline & S. meridionalis & 382 & & & \\
\hline & Total & 16.718 & 7 & 0,62 & 0,18 \\
\hline \multirow{4}{*}{ QUEQUEN SALADO } & H. parchappii & 1.932 & & & \\
\hline & M. brasiliensis & 4 & & & \\
\hline & S. meridionalis & 4.237 & & & \\
\hline & Total & 6.173 & 3 & 0,23 & 0,27 \\
\hline \multirow{5}{*}{ CAMPO LABORDE } & H. parchappii & 10 & & & \\
\hline & B. parchappii & 28 & & & \\
\hline & M. brasiliensis & 2 & & & \\
\hline & S. merdionalis & 1.632 & & & \\
\hline & Total & 1.672 & 4 & 0,40 & 0,06 \\
\hline \multirow{12}{*}{ CALERA } & H. parchappii & 49 & & & \\
\hline & A. minor & 203 & & & \\
\hline & D. kermatoides & 4.319 & & & \\
\hline & B. peregrina & 1.187 & & & \\
\hline & A. nordestensis & 31 & & & \\
\hline & U. concentricus & 3 & & & \\
\hline & R. reticulatus & 12.112 & & & \\
\hline & M. brasiliensis & 942 & & & \\
\hline & S. meridionalis & 224 & & & \\
\hline & O. unguis & 15 & & & \\
\hline & Pisidium sp. & 613 & & & \\
\hline & Total & 19.698 & 11 & 1,01 & 0,51 \\
\hline
\end{tabular}


(Figuras 2, 6A,C; Tabla 2). Desde el Holoceno temprano (ca. 10,4 ka AP; Figuras 6A,C) hasta 9,6 ka AP se infiere la formación de ambientes palustres y desecación de los mismos en Paso Otero 5. A escala geográfica de la cuenca media del río Quequén Grande se registra un incremento de los taxones higrófilos (i.e. Succinea meridionalis), probablemente como respuesta a un aumento de la humedad durante el Holoceno temprano en Paso Otero 1 y 5. (Figura 6A; Tablas 3,4). El desarrollo de microambientes pantanosos está avalado, además, por datos provenientes de dos perfiles realizados en el curso inferior del río Quequén Salado (Steffan et al., 2010), los que indican condiciones pantanosas en la base del Miembro Río Salado. Del mismo modo, en ambientes de llanura alejados del curso fluvial Tapalqué, como el sitio Campo Laborde, estas condiciones pantanosas se extienden hasta los $c a .8$ ka AP (Figura 6C). En consecuencia, el sector noroeste del área Interserrana (cuenca superior del arroyo Tapalqué) muestra paleoambientes coincidentes con los contextos aproximadamente sincrónicos del sudeste del área Interserrana. Más aún, incluso estadísticamente, a través del análisis de coordenadas principales (PCA, Principal Coordinate Analysis), es posible observar la superposición espacial entre los sitios Paso Otero 5 y Campo Laborde (Figura 7B). Nuestros resultados son coincidentes con interpretaciones paleoambientales para la región basados en otras fuentes de evidencia. Específicamente, los estudios basados en el polen y la fauna de vertebrados (e.g. Prado et al., 2001; Grill et al., 2007) registran cambios hacia una vegetación de pantanos y de planicies de inundación $c a$. 10,4 ka AP y también con aquellos que sugieren condiciones subhúmedas-secas para el centro de la región Pampeana (e.g. Prieto, 1996; Zárate et al., 2000; Quattrocchio et al., 2008). Desde el punto de vista de las investigaciones arqueológicas (Martínez 1999; Messineo \& Politis, 2007) la malacofauna apoya la hipótesis de que la presencia de la megafauna se recupera en contextos ambientales pantanosos.

\section{Variaciones de la malacofauna durante el Holoceno medio (ca. 6,5-3,5 ka AP): Paso Otero 1, Paso Otero 3, Paso Otero 5, Quequén Salado}

El registro malacológico del Holoceno medio (Figuras 6a,b) podría ser diferenciado cronológicamente en dos etapas: la primera, (ca. 6,5 ka AP y ca. $5 \mathrm{ka} \mathrm{AP}$ ), caracterizada por una escasa diversidad de moluscos (Paso Otero 1 y 3 ) (Figuras 2,6A); y la segunda (5 ka AP y 3,5 ka AP), por una mayor diversidad (Paso Otero 1, 3, 5). El incremento de moluscos fluvio-lacustres (e.g. Heleobia parchappii, Biomphalaria peregrina) se registra con posterioridad a la máxima transgresión marina ocurrida sobre la desembocadura del río Quequén Grande entre 7,6 ka AP y ca. 5,3 ka AP (Ferrero, 1996). El máximo transgresivo del Holoceno se produjo como consecuencia del incremento de la temperatura ocurrido durante el Optimo Climático o Máximo Termal del Holoceno medio (e.g. Schnack et al., 2005; Briner et al., 2006). En Paso Otero 1 - con mayor intensidad - y Paso Otero 3 - con menor expresión -, la presencia de discordancias erosivas - rasgos de canal y la fragmentación observada en la malacofauna y restos óseos (ver Kaufmann \& Gutiérrez, 2004) indican la acción de un flujo de mayor energía, relacionado con eventos de inundación ( $c a$. 4,9-3 ka AP). Al mismo tiempo, este evento pudo haber generado una sobre representación de ambos taxones en la muestra. Adicionalmente a la evidencia presentada, la similitud estadística en la composición taxonómica entre ambos sitios, sugieren un evento de mayor humedad entre $c a .4,9$ ka AP y $c a .4,2$ ka $\mathrm{AP}$, vinculado probablemente con un aumento en el patrón de precipitaciones, registrado previamente por Bonadonna et al. (1999) para el área sobre la base de variaciones en la composición isotópica de conchillas de moluscos. Dicho escenario pudo haber favorecido la formación de sectores lacustres locales, aún dentro de una tendencia generalizada hacia condiciones generales más secas (Tonni et al., 1999). Asimismo, han sido registradas condiciones de mayor humedad en la cuenca media del río Quequén Grande a partir del desarrollo de horizontes de paleosuelos reconocidos en estudios geoarqueológicos (Favier Dubois, 2006). En el área Quequén Salado podría ser sincrónico, además, con el evento húmedo documentado por registros polínicos del sudeste bonaerense (Sauce Grande, ca. 5 ka AP) (Quattrocchio \& Borromei, 1998). La tendencia hacia condiciones más secas señalada previamente por Tonni et al., (1999) se evidencia en el río Quequén Grande y río Quequén Salado señalada por la desecación o exposición subaérea de los depósitos lacustres, inferida a partir de la disminución de los taxones acuáticos. En este trabajo y para el mismo período (4,9-3 ka AP), los resultados basados en variaciones de $\delta{ }^{18} \mathrm{O}$ sobre conchillas de $H$. parchappii y la presencia de Retidiscus reticulatus, taxón típico del Dominio Subtropical y actualmente viviente exclusivamente hasta el sudeste de Brasil, permiten corroborar un incremento de la temperatura en los perfiles de los sitios Paso Otero 1 y 3 (ca. 4,9-3 ka AP) (Steffan, 2009).

\section{Variaciones de la malacofauna durante el Holoceno tardío (ca. 3,5-500 años AP): Paso Otero 1, Paso Otero 3, Paso Otero 5, Cortaderas, Quequén Salado y Calera}

De acuerdo con hipótesis paleoclimáticas generales basadas en datos de vertebrados, polen e isótopos estables sobre moluscos continentales de la región Pampeana (Prieto, 1996; Bonadonna et al., 1999; Tonni et al., 1999; Quattrocchio et al., 2008), el inicio del Holoceno tardío se caracteriza por una tendencia de condiciones semiáridas con alternancia de eventos húmedos cortos. En líneas generales nuestras evidencias malacológicas avalan dicho patrón para los sitios analizados del sudeste del Área Interserrana en los perfiles de Paso Otero 1 y 3 del río Quequén Grande, en los que se registra un cambio de condiciones lacustres hacia desecación ca. 3 ka AP(Figuras 6A-C). Por el contrario, en el norte del área Interserrana (sistema serrano) la malacofauna sugiere condiciones lacustres vinculadas con condiciones más húmedas que las actuales ( $c a$. 3-2 ka AP) (Figura 6C, Tabla 2) tal como se observa en el Sitio Calera. El cual se encuentra poco relacionado estadísticamente con los sitios restantes (Figuras 7A,B).

En términos generales, la malacofauna indica eventos húmedos con fechados similares para la cuenca media del río 


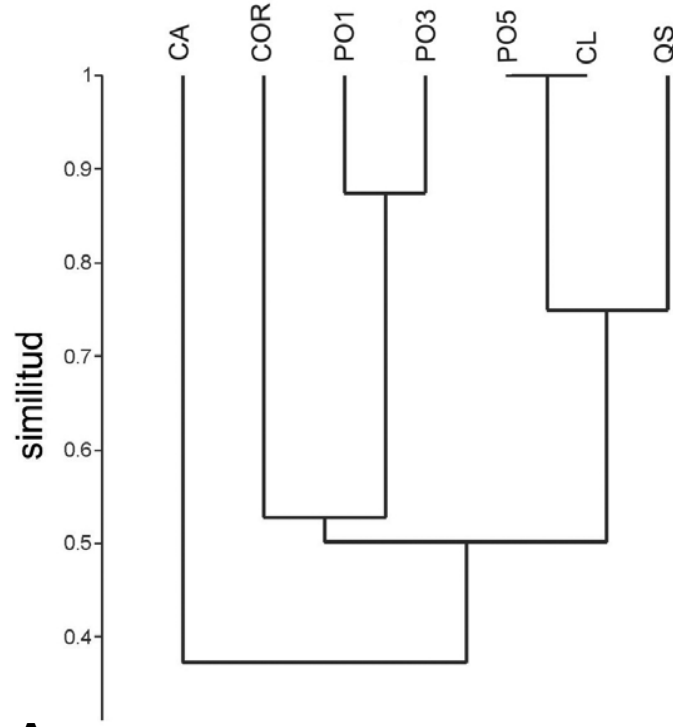

A

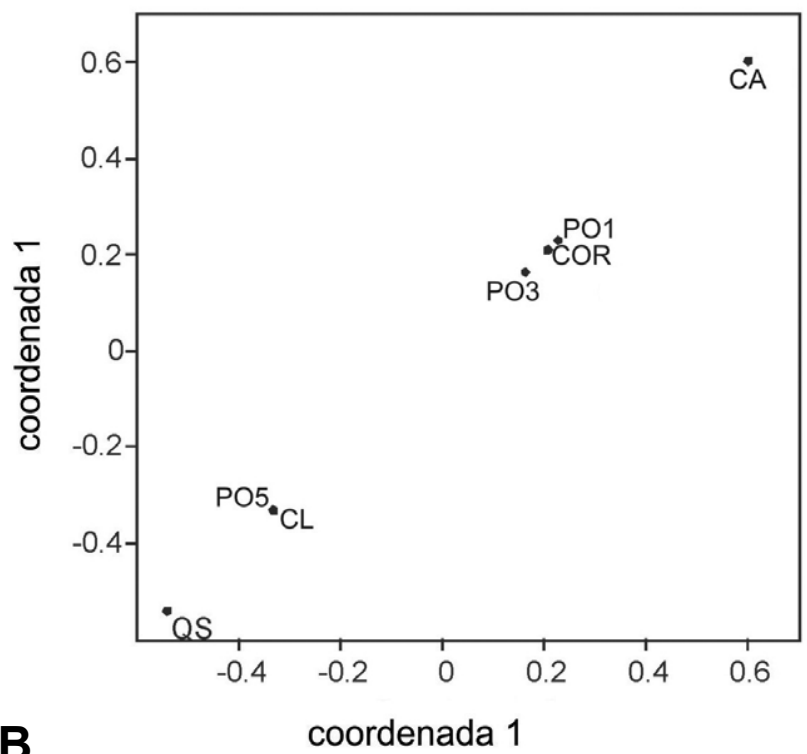

Figura 7. Análisis multivariados de la malacofauna recuperada en los sitios arqueológicos. A, cluster Analysis (CA). Dendograma del Coeficiente de Asociación de Jaccard -CAJ- de las especies de moluscos analizados en los sitios: PO1 = Paso Otero 1; PO3 = Paso Otero 3; PO5 = Paso Otero 5; CL = Campo Laborde; COR = Cortaderas; $\mathbf{Q S}=$ Quequén Salado; $\mathbf{C A}=$ Calera. $\mathbf{r}=$ coeficiente de correlación cofenética. $\mathbf{B}$, análisis de Coordenadas Principales (PCA) de las especies presentes en los sitios.

Figure 7. A, Cluster analysis based on Jaccard -CAJ- association coefficient measures of species of mollusks analyzed in sites. $\mathbf{r}=$ cophenetic correlation coefficient. B, principal Coordinates Analysis (PCA) of species inhabited in the sites.

Quequén Grande (Paso Otero 5, ca. 2,5 ka AP; Paso Otero 3, ca. $2,2 \mathrm{ka} \mathrm{AP-2} \mathrm{ka}$ AP), en Cortaderas (con posterioridad a $\mathrm{ca}$. 2,2 ka AP) y en el sitio Calera (ca. 2 ka AP). La abundancia de taxones fluvio-lacustres y el alto grado de fragmentación y corrasión observado en las conchillas sugieren que al menos algunas de estas fueron afectadas por una corriente de mayor energía, como resultado de eventos de inundación acotados (ca. 2,2-2 ka AP). Dichos eventos húmedos podrían exceder la escala local y ser algún fenómeno a escala regional. Sin embargo, los análisis malacológicos en otros sitios del área con similares fechados permitirán contrastar las tendencias observadas en este trabajo.

Posteriormente a dichos eventos húmedos se infiere predominancia de microambientes encharcados temporarios y su posterior desecación en Paso Otero 3 y Cortaderas (Figuras 5a,b). A pesar de la proximidad temporal de las características paleoambientales entre Paso Otero 3 y Cortaderas, el coeficiente de Jaccard no muestra una marcada similitud entre ambos (Figura 7A), lo cual se explicaría porque precisamente, con excepción del mencionado evento húmedo, cada microambiente presenta su propia dinámica y responde particularmente a las variaciones climáticas, tal como ocurre en la actualidad. Hacia el final del Holoceno tardío y durante épocas históricas, la evidencia malacológica de los perfiles analizados en la cuenca superior del río Quequén Salado indica condiciones palustres entre $c a$. 1 ka AP y 800 años AP. Hacia $c a .360$ años AP se registran condiciones semiáridas (Steffan et al., 2010).

Los cambios documentados para el Holoceno en el patrón de distribución de los moluscos (e.g. Retidiscus) y el incremento de abundancia relativa de taxones acuáticos sugieren cambios de la humedad sincrónicos en diferentes lugares del paisaje del área Interserrana. Dichas condiciones pudieron potencialmente afectar el patrón de distribución de las poblaciones humanas de cazadores-recolectores, sin embargo su análisis no es objeto del presente estudio. No obstante los resultados aquí obtenidos ofrecen información contextual para la interpretación de sus pautas adaptativas reconocidas en los sitios arqueológicos analizados. En este sentido, las inferencias paleoambientales avalan una mayor diversidad de microambientes lacustres en sectores de valles y divisorias para el Holoceno tardío $(\sim 3$ y $2 \mathrm{ka}$ ), lo cual habría favorecido la proliferación de aves y especies de afinidad acuática. Estas características habrían contribuido a una mayor diversificación de los recursos disponibles. Dicho escenario es coincidente con una mayor abundancia taxonómica en el registro zooarqueológico del área (Politis et al., 2005; Martínez, 2006; Massigoge, 2006).

\section{CONCLUSIONES}

Los datos obtenidos permiten establecer los primeros registros fósiles ilustrados de los siguientes taxones para el Holoceno del área Interserrana: Uncancylus concentricus, "Aplexa" minor, Drepanotrema kermatoides, Antillorbis nordestensis, Omalonyx unguis y del bivalvo Pisidium sp. La mayoría de ellos se encuentran vivientes en el área relevada, excepto Lymnaea viator, Zilchogyra costellata y Retidiscus reticulatus, aunque no se descarta su aparición en futuros estudios. Z . costellata y $R$. reticulatus serían indicativos de condiciones de mayor humedad que en la actualidad. 
Conjuntamente, cuali y cuantitativamente, la malacofauna del área Interserrana analizada en este trabajo sugiere, para distintos momentos, las siguientes condiciones paleoambientales: (i) Holoceno temprano ( $c a .10,4-6,5 \mathrm{kaAP}$ ): características palustres seguidas por un leve descenso de la humedad. Interrumpidas por dos eventos breves de mayor humedad en $c a$. 8,8 ka AP y $c a$. 7,8 ka AP; (ii) Holoceno medio ( $c a$. 6,5-3,5 ka AP): incremento en el patrón de precipitaciones hacia fines del Holoceno medio el $c a$. 4,94,7 ka AP, ocurrencia del Optimo Climático y su efecto biogeográfico en ambiente continental; (iii) Holoceno tardío (ca. 3,5 ka AP-500 años AP): inicialmente tendencia de condiciones semiáridas con alternancia de eventos húmedos cortos; entre $c a .3$ a 2 ka AP condiciones más húmedas que las actuales en el área, específicamente Calera, Cortaderas, Paso Otero 3 y Paso Otero 5 vinculadas con alteración del patrón de precipitaciones. Considerable variabilidad ambiental ligada a eventos locales de inundación y evaporación entre $c a$. 2,2-1,5 ka AP. Desde $c a$. 1,5 ka AP, formación de sectores encharcados hacia ambientes lacustres someros en $c a$. 960 años AP, con una desecación de los mismos hacia $c a .790$ años AP.

En la agenda futura, las conclusiones de este estudio deberán ser contrastadas y discutidas con datos isotópicos del río Quequén Grande. Asimismo, un estudio detallado de la malacofauna moderna complementado con datos de la geoquímica del suelo y del agua permitirá profundizar el conocimiento de los aspectos ecológicos.

\section{AGRADECIMIENTOS}

A G. Politis, P. Messineo, G. Martínez, P. Madrid y A. Massigoge por brindar información de los sitios arqueológicos analizados. AA. Tablado (MACN), C. Damborenea y M. Tassara (FCNYM) por su asesoramiento en la consulta de colecciones de referencia. A D-D. Rousseau por su asesoramiento en relación con el análisis de la malacofauna. A C.F. Dubois por su colaboración. A J.L. Prado por su colaboración con los análisis estadísticos. Este artículo es una producción de la Unidad Ejecutora INCUAPA-CONICET (Investigaciones Arqueológicas y Paleontológicas del Cuaternario Pampeano) dirigido por el Gustavo Politis y el J.L. Prado. Al PICT 468 y PIP 2010-0080. PIP (2010 0080; 2013 00372) y 11/N726.

\section{REFERENCIAS}

Aguirre, M.L. 1990. Holocene macrobenthic molluscan associations from north-eastern Buenos Aires Province, Argentina. Quaternary of South America and Antarctic Peninsula, 7:161195.

Aguirre, M.L. \& Farinati, E.A. 2000. Aspectos sistemáticos, de distribución y paleoambientales de Littoridina australis (D’Orbigny, 1835) (Mesogastropoda) en el Cuaternario marino de Argentina (Sudamérica). Geobios, 33:569-597.

Aguirre, M.L.; Miquel, S.E.; González, C.A.; Kröhling, D.; Zucol, A.F.; Brea, M. \& Eastoe, C. 2011. Malacofauna continental holocena y paleoambientes en Villa Valle María (Diamante, Entre Ríos, Argentina). Geobios, 44:1-17. doi:10.1016/j. geobios.2010.06.003
Aramayo, S.; Schillizzi, R. \& Gutiérrez Téllez, B. 2002. Evolución paleoambiental del Cuaternario en la costa atlántica del sur de la provincia de Buenos Aires, Argentina. Revista de la Sociedad Geológica de España, 15:95-104.

Bonadonna, F.P.; Leone, G. \& Zanchetta, G. 1999. Stable isotopes analysis on the last $30 \mathrm{ka}$ molluscan fauna from Pampa Grassland, Bonaerense region, Argentina. Palaeogeography, Palaeoclimatology, Palaeoecology, 153:289-308. doi: 10.1016/ s0031-0182(99)00063-2

Bonetto, A.A.; Rumi, A. \& Tassara, M.P. 1990. Notas sobre el conocimiento limnológico de los gasterópodos paranaense y sus relaciones tróficas. II. Planorbidae, con aspectos distribucionales y sanitarios. Ecosur, 6:69-84.

Bonomo, M.; Leon, D.C.; Osterrieth, M.; Steffan, P. \& Borelli, N. 2013. Paleoenvironmental studies of Alfar archaeological site (Southeastern Pampas, Argentina): phytoliths, gastropods and archaeofauna. Quaternary International, 287:34-46. doi:10.1016/j.quaint.2012.03.039

Bouchet, P. \& J.-P. Rocroi, 2005. Classification and nomenclator of gastropod families. Malacologia, 47:1-397.

Briner, J.P.; Michelutti, N.; Francis, D.R.; Miller, G.H.; Axford,Y.; Wooller, M.J. \& Wolfe, A.P. 2006. A multi-proxy lacustrine record of Holocene climate change on northeastern Baffin Island, Arctic Canada. Quaternary Research, 65:431-442. doi: 10.1016/j.yqres.2005.10.005

Cabrera, A.L. \& Willink, A. 1973. Biogeografia de América Latina. Washington, OEA, 120 p. (Serie de Biologia 13).

Castellanos, Z.J.A. \& Miquel, S.E. 1991. Distribución de los Pulmonata Basommatophora. In: R.A. Ringuelet \& Z.J.A. Castellanos (eds.) Fauna de agua dulce de la República Argentina, Fundación para la Educación, la Ciencia y la Cultura, p. 1-11.

Castillo, C.; Martín-González, E.; Yanes, Y.; Ibáñez, M.; De La Nuez, J.; Alonso, M.R. \& Quesada, M.L. 2002. Estudio preliminar de los depósitos dunares de los Islotes del Norte de Lanzarote. Implicaciones paleoambientales. Geogaceta, 32:79-82.

Cazzaniga, N.J. 1982. Notas sobre hidróbidos argentinos II (Gastropoda Rissoidea). Una Littoridina del 'grupo parchappii' en Península Valdés (Chubut). Revista del Museo de La Plata, Sección Zoología, 129:11-16.

Cheetham, A.H. \& Hazel, L.E. 1969. Binary (presence-absence) similarity efficient. Journal of Paleontology, 43:1130-1136.

De Francesco, C. \& Isla, F. 2004. Reproductive period and growth rate of the freshwater snail Heleobia parchappii (d'Orbigny, 1835) (Gastropoda: Rissooidea) in a shallow brackish habitat Buenos Aires Province, Argentina. Malacologia, 45:443-450.

De Francesco, C. \& Dieguez, S. 2006. Paleoambientes del Cuaternario tardío del sur de Mendoza: estado del conocimiento, problemas y perspectivas. In: V. Durán \& V. Cortegoso (eds.) Arqueología y ambientes de áreas naturales protegidas de la provincia de Mendoza, Universidad Nacional de Cuyo/Facultad de Filosofía y Letras, p. 69-80.

De Francesco, C.G. \& Hassan, G.S. 2009. The significance of molluscs as paleoecological indicators of freshwater systems in central-western Argentina. Palaeogeography, Palaeoclimatology, Palaeoecology, 274:105-113. doi:10.1016/j.palaeo.2009.01.003

De Francesco, C.; Zárate, M. \& Miquel, S. 2007. Late Pleistocene mollusc assemblages and inferred paleoenvironments from the Andean piedmont of Mendoza, Argentina. Palaeogeography, Palaeoclimatology, Palaeoecology, 251:461-469. doi:10.1016/j. palaeo.2007.04.011 
Dimieri, L.; Delpino, S. \& Turienzo, M. 2005. Estructura de las Sierras Australes de Buenos Aires. In: R.E. Barrio; R.O. Etcheverry; M.F. Caballé \& E. Llambías (eds.) Geología y Recursos Minerales de la Provincia de Buenos Aires, Relatorio del XVI Congreso Geológico Argentino, p. 101-118.

d'Orbigny, A. 1835. Synopsis terrestrium et fluviatilium molluscorum, in suo per Americam meridionalem itinere. Magasin de Zoology, 5:23-44.

Favier Dubois, C. 2006. Dinámica fluvial, paleoambientes y ocupaciones humanas en la localidad Paso Otero, Río Quequén Grande, provincia de Buenos Aires. Intersecciones en Antropología, 7:109-127.

Fernández, D. 1973. Catálogo de la malacofauna terrestre argentina. La Plata, Comisión de Investigaciones Científicas (CIC), 197 p. (Monografías 4).

Fernández, D. \& Castellanos, Z.J.A. de. 1973. Clave genérica de la malacofauna terrestre Argentina. Revista del Museo de La Plata (Nueva Serie), Sección Zoología, 11: 265-285.

Ferrero, L. 1996. Paleoecología de ostrácodos holocenos del estuario del río Quequén Grande (Provincia de Buenos Aires). Ameghiniana, 33:209-222.

Fidalgo, F.; De Francesco, F. \& Colado, U. 1973. Geología superficial de las hojas Castelli, J.M. Cobo y Monasterio, Provincia de Buenos Aires. In: CONGRESO GEOLÓGICO ARGENTINO, 5, 1973. Actas, Carlos Paz, p. 27-39.

Fidalgo, F.; Gentile, R. \& Correa, H. 1986. Geología y geomorfología en la cuenca del arroyo Tapalqué. La Plata, Comisión de Investigaciones Científicas (CIC), 73 p. (Informe 30).

Figini, A.; Huarte, R.; Carbonari, J. \& Tonni, E. 1998. Edades C-14 en un perfil del arroyo Tapalqué, provincia de Buenos Aires, Argentina. Contribución a la cronología de acontecimientos faunístico-ambientales. In: CONGRESO LATINOAMERICANO DE GEOLOGÍA Y CONGRESO NACIONAL DE GEOLOGÍA ECONÓMICA, 10, 1998. Actas, Buenos Aires, p. 27-31.

Fonseca, A.L.M. \& Thomé, J.W. 1995. A new genus and species of Charopidae (Gastropoda, Endodontoidea, Stylommatophora), Retidiscus reticulatus from Southern Brazil. Walkerana, 7:55-61.

Frenguelli, J. 1950. Rasgos generales de la hidrografía de la Provincia de Buenos Aires. LEMIT, 72 p.

Fucks, E.; Aguirre, M. \& Deschamps, C. 2005. Late Quaternary continental and marine sediments at northeastern bonaerensian area (Argentina): fossil content and palaeoenvironmental interpretation. Journal of South American Earth Sciences, 20:57-64. doi:10.1016/j.jsames.2005.05.003

Gaillard, C. \& Castellanos, Z.A. 1976. Mollusca Gasteropoda Hydrobiidae. In: R.A. Ringuelet \& Z.J.A. Castellanos (eds.) Fauna de agua dulce de la República Argentina, Fundación para la Educación, la Ciencia y la Cultura, p. 1-40.

Gower, J.C. 1966. Some distance properties of latent root and vector methods used in multivariate analysis. Biometrika, 53:325-338.

Grill, S.; Borromei, A.M.; Martínez, G.; Gutiérrez, M.; Cornou, M.E. \& Olivera, D. 2007. Palynofacial analysis in alkaline soils - Paso Otero 5 archaeological site (Necochea district, Buenos Aires province, Argentina). Journal of South American Earth Sciences, 24:34-47. doi:10.1016/j.jsames.2007.02.010

Gutiérrez Grégoric, D.E.; Núñez, V.; Rumi, A. \& Roche, M.A. 2006. Freshwater gastropods from Plata Basin, Argentina, checklist and new locality records. Comunicaciones de la Sociedad Malacolológica de Uruguay, 9:51-60.

Gutiérrez, M.A.; Martínez, G.; Luchsinger, H.; Grill, S.; Zucol, A.F.; Hassan, G.S.; Barros, M.P.; Kaufmann, C.A. \& Álvarez, M.C. 2011. Paleoenvironments in the Paso Otero locality during
Late Pleistocene-Holocene (Pampean region, Argentina): an interdisciplinary approach. Quaternary International, 245:37-47. doi: 10.1016/j.quaint.2010.11.010

Hammer, Ø.; Harper, D.A.T. \& Ryan, P.D. 2001. PAST: paleontological statistics software package for education and data analysis. Palaeontologia Electronica, 4. Avaliable at http://palaeo-electronica. org/2001_1/past/issue1_01.htm; accessed on 07/04/2014.

Holliday, V.; Martínez, G.; Johnson, E. \& Buchanan, B. 2003. Geoarchaeology of Paso Otero 5 (Pampas of Argentina). In: L. Miotti; M. Salemme \& N. Flegenheimer (eds.) Ancient evidences for paleo south americans: from where the south winds blow, Center for the Studies of the First Americans (CSFA) and Texas A \& M University Press, p. 37-43.

Ituarte, C.F. 1996. Argentine species of Pisidium Pfeiffer, 1821, and Musculium Link, 1807 (Bivalvia: Spahaeriidae). Veliger, 39:189-203.

Johnson, E.; Politis, G.; Martínez, G.; Hartwell, W.; Gutiérrez, M. \& Hass, H. 1998. The radiocarbon chronology of Paso Otero 1 in the Pampean Region of Argentina. Quaternary of South America and Antarctic Peninsula, 11:15-25.

Kaufmann, C. \& Gutiérrez, M.A. 2004. Dispersión potencial de huesos de guanaco en medios fluviales y lacustres. In: G. Martínez; M. Gutiérrez; R. Curtoni; M. Berón \& P. Madrid (eds.) Aproximaciones contemporáneas a la arqueología pampeana. Perspectivas teóricas, metodológicas, analíticas y casos de estudio, Facultad de Ciencias Sociales/UNCPBA, p. 129-146.

Kotzian, C.B. \& Simões, M.G. 2006. Taphonomy of recent freshwater molluscan death assemblages, Touro Passo Stream, southern Brazil. Revista Brasileira de Paleontologia, 9:243-260.

Landoni, N. 1992. Inventario de los moluscos de agua dulce de la Provincia de Buenos Aires. In: H. Lopez \& E.P. Tonni (eds.) Situación ambiental de la provincia de Buenos Aires. Recursos $y$ rasgos naturales en la evaluación ambiental, Comisión de Investigaciones Científicas, p. 3-57.

Lucena, D.T. 1954. Tropicorbis nordestensis n. sp. do nordeste do Brasil. Revista Brasileira de Malariologia e Doenças Tropicais, 6:329-331.

Madrid, P.; Politis, G.; March, R. \& Bonomo, M. 2002. Arqueología microregional en el sudoeste de la región Pampeana Argentina: el curso del río Quequén Salado. Relaciones, 27:327-355.

Martínez, G. 1999. Tecnologia, subsistencia y asentamiento en el curso medio del Río Quequén Grande: un enfoque arqueológico. Facultad de Ciencias Naturales y Museo, Universidad Nacional de La Plata, Ph.D. thesis, 185 p.

Martínez, G. 2006. Arqueología del curso medio del Río Quequén Grande: estado actual y aportes a la arqueología de la región pampeana. Relaciones, 31:249-275.

Massigoge, A. 2007. Procesos de formación del registro arqueológico en el sitio Cortaderas (partido de San Cayetano, provincia de Buenos Aires). Intersecciones en Antropología, 8:197-214.

Messineo, P.G. \& Politis, G. 2007. El sitio Calera. Un depósito ritual en las Sierras Bayas (Sector Noroccidental de Tandilia). In: C. Bayón; A. Pupio \& M.I. González (eds.) Arqueología de las Pampas, Sociedad Argentina de Antropología, p. 697-720.

Miquel, S.E. \& Aguirre, M. 2011. Taxonomía de los gasterópodos terrestres del Cuaternario de Argentina. Revista Española de Paleontología, 26:101-133.

Miquel, S.E.; Ramírez. R. \& Thomé, J.W. 2004. Preliminar list of the Punctoidea from the State of Rio Grande do Sul, Brazil, with description of two new species (Mollusca, Gastropoda, Stylommatophora) (in spanish). Revista Brasileira Zoologia, 21:925-935. doi: 10.1590/S0101-81752004000400030 
Miquel, S.E. \& Steffan, P.G., 2005. Hallazgo de Retidiscus reticulatus (Gastropoda, Pulmonata, Charopidae) en la Argentina, un posible indicador de paleoclima húmedo Holoceno en el sur bonaerense. Ameghiniana, 42:495-500.

Moine, O.; Rousseau, D-D. \& Antoine, P. 2008. The impact of Dansgaard-Oeschger cycles on the loessic environment and malacofauna of Nussloch (Germany) during the Upper Weichselian. Quaternary Research, 70:91-104. doi: 10.1016/j. yqres.2008.02.010

Mormeneo, L. 1992. Caracterización de sedimentos en dos perfiles geológicos del río Quequén Salado, provincia de Buenos Aires. In: JORNADAS GEOLÓGICAS BONAERENSES, 3, 1992. Actas, La Plata, p. 249-251.

Naranjo-García, E. \& Gómez Espinosa, C. 2004. Moluscos. In: F. Bautista-Zúñiga; H. Delfín-González \& J.L. Palacio Prieto (eds.) Técnicas de muestreo para manejadores de recursos naturales, Universidad Nacional Autónoma de México/Universidad Autónoma de Yucatán/CONACYT/INE, p. 211-233.

Osterrieth, M.; Martínez, G.; Gutiérrez, M. \& Alvarez, F. 2008. Biomorfos de sílice en secuencias pedoarqueológicas del sitio Paso Otero 5, Buenos Aires, Argentina. In: M.A. Korstanje \& M.P. Babot (eds.) Matices interdisciplinarios en estudios fitoliticos y de otros microfósiles, BAR International S1870, p. $77-90$.

Politis, G. 1984. Climatic variations during historical times in eastern Buenos Aires Pampas, Argentina. Quaternary of South America and Antarctic Peninsula, 3:133-161.

Politis, G.G. \& Messineo, P.G. 2008. The Campo Laborde site: new evidence for the Holocene survival of Pleistocene megafauna in the Argentine Pampas. Quaternary International, 191:98-114. doi:10.1016/j.quaint.2007.12.003

Politis, G.G.; Messineo, P.G.; Kaufmann, C.A.; Barros, M.P.; Alvarez, M.C.; Di Prado, V. \& Scalise, R. 2005. Persistencia ritual entre cazadores recolectores de la llanura pampeana. In: P. Kaulicke \& T. Dillehay (eds.) Encuentros: identidad, poder y manejo de los espacios públicos, Lima, Pontificia Universidad Católica del Perú, p. 67-90 (Boletín de Arqueología PUCP 9).

Politis, G.G.; Tonni, E. \& Fidalgo, F. 1983. Cambios corológicos de algunos mamíferos en área interserrana de la provincia de Buenos Aires durante el Holoceno. Ameghiniana, 20:72-80.

Prado, J.L.; Alberdi, M.T.; Azanza, B. \& Sánchez, B. 2001. Climate and changes in mammal diversity during the Late Pleistocene-Holocene in the Pampean Region (Argentina). Acta Paleontologica Polonica, 46:261-276.

Prado, J.L.; Alberdi, M.; Martínez, G. \& Gutiérrez, M. 2005. Equus (Amerhippus) neogeus Lund 1840 (Equidae, Perissodactyla) at Paso Otero 5 site (Argentina): its implication for the horses extinction. Neues Jahrbuch für Geologie und Paläontologie, 8:449-468.

Prieto, A. 1996. Late Quaternary vegetation and climatic changes in the pampa grassland of Argentina. Quaternary Research, 45:75-88. doi:10.1006/qres. 1996.0007

Prieto, A.; Blasi, A.; De Francesco, C. \& Fernández, C. 2004. Environmental history since $11,000{ }^{14} \mathrm{C}$ yr B.P. of the northeastern pampas, Argentina, from alluvial sequences of the Luján River. Quaternary Research, 62:146-161. doi:10.1016/j. yqres.2004.04.006

Quattrocchio, M.E. \& Borromei A.M. 1998. Paleovegetational and paleoclimatic changes during the Late Quaternary in southwestern Buenos Aires province and southern Tierra del Fuego (Argentina). Palynology, 22:67-82. doi:10.1080/01916 122.1998.9989503
Quattrocchio, M.E.; Borromei, A.M.; Deschamps, C.M.; Grill S.C. \& Zavala, C.A. 2008. Landscape evolution and climate changes in the Late Pleistocene-Holocene, southern Pampa (Argentina): evidence from palynology, mammals and sedimentology. Quaternary International, 181:123-138. doi:10.1016/j. quaint.2007.02.018

Reimer, P.J et al. 2013. IntCal13 and MARINE13 radiocarbon age calibration curves 0-50,000 years cal BP. Radiocarbon, 55:18691887. doi: 10.2458/azu_js_rc.55.16947

Ringuelet, R.A. 1955. Panorama zoogeográfico de la provincia de Buenos Aires. Notas del Museo de La Plata, 18:1-45.

Ringuelet, R.A. 1961. Rasgos de la zoogeografía de la Argentina. Physis, 22:151-170.

Rohlf, F.J. 1970. Adaptive hierarchical clustering schemes. Systematic Zoology, 19:58-82.

Rousseau, D-D.; Gerasimenko, N.; Matviischina Z. \& Kukla, G. 2001. Late Pleistocene environments of the central Ukraine. Quaternary Research, 56:349-356. doi:10.1006/qres.2001.2270

Rousseau, D-D.; Hatte, C.; Guiot, D.; Duzer, P.; Schevin, P. \& Kukla, G. 2006. Inversing biomes and $\delta{ }^{13} \mathrm{C}$ for the Grande Pile Eemian record: reconstruction of an instable climate interval. Quaternary Science Reviews, 25:2806-2819. doi:10.1016/j. quascirev.2006.06.011

Rumi, A. 1991. La familia Planorbidae Rafinisque, 1815 en la República Argentina. In: R.A. Ringuelet \& Z.J.A. Castellanos (eds.) Fauna de agua dulce de la República Argentina, Fundación para la Educación, la Ciencia y la Cultura, p. 5-51.

Rumi, A.; Gregoric, D.E. \& Roche, M.A. 2007. Growth rate fitting using the von Bertalanffy model: analysis of natural populations of Drepanotrema spp. snails (Gastropoda: Planorbidae). Revista de Biología Tropical, 55:559-567.

Scarabino, F. 2004. Lista sistemática de los Gastropoda dulciacuícola vivientes de Uruguay. Comunicaciones de la Sociedad Malacológica del Uruguay, 8:347-356.

Schnack, E.; Isla, F.; De Francesco, F. \& Fucks, E. 2005. Estratigrafía del Cuaternario marino tardío en la provincia de Buenos Aires. In: CONGRESO GEOLÓGICO ARGENTINO, 16, 2005. Relatorio, Buenos Aires, p. 159-182.

Simone, L.R.L. 2006. Land and freshwater mollusks of Brazil: an illustrated inventory of the Brazilian malacofauna, including neighboring regions of South America, respect to the terrestrial and freshwater ecosystems. São Paulo, EGB Fapesp, 390 p.

Steffan, P.G. 2007. Análisis paleoambiental en base al estudio malacológico del Sitio Paso Otero 1 (Región Pampeana, Argentina). Intersecciones en Antropología, 8:173-184.

Steffan, P.G., 2009. Reconstrucción paleoambiental de contextos arqueológicos del área interserrana. Facultad de Ciencias Naturales y Museo de la Universidad Nacional de La Plata, Ph.D. thesis, 414 p.

Steffan, P.G.; Madrid, P.; Gómez G. \& Mormeneo, L. 2010. Evaluación de la información paleoambiental y la dinámica de ocupación humana en el curso inferior del río Quequén Salado durante el holoceno tardío. In: M. Berón; L. Luna; M. Bonomo; C. Montalvo; C. Aranda \& M. Carrera Aizpitarte (eds.) Mamül Mapu: pasado y presente desde la arqueología pampeana, Editorial Libros del Espinillo, p. 215-226.

Stimpson, W. 1865. Researches upon the Hydrobiinae and allied forms chiefly made upon materials in the museum of the Smithsonian Institution. Smithsonian Miscellaneous Collections, 7:1-59.

Teruggi, M.E. \& Kilmurray, J.O. 1980. Sierras septentrionales de la provincia de Buenos Aires. In: J.C.M. Turner (ed.) Geología Regional Argentina, Academia Nacional de Ciencias, p. 919-956. 
Tonni, E.P.; Cione, A. \& Figini. A. 1999. Predominance of arid climates indicated by mammals in the pampas of Argentina during the late Pleistocene and Holocene. Palaeogeography, Palaeoclimatology, Palaeoecology, 147:257-281. doi: 10.1016/ s0031-0182(98)00140-0

Tonni, E.P.; Cione, A.L. \& Figini, A. 2001. Chronology of Holocene pedogenetic events in the Pampean area of Argentina. Current Research in the Pleistocene, 18:124-127.
Zárate, M.A.; Kemp, R.; Espinosa, M. \& Ferrero, L. 2000. Pedosedimentary and palaeoenvironmental significance of a Holocene alluvial sequence in the southern Pampas, Argentina. The Holocene, 10:481-488. doi: 10.1191/095968300669846317

Received in July, 2013; accepted in April, 2014. 